\title{
A Rate-Distortion Approach to Index Coding
}

\author{
Sinem Unal and Aaron B. Wagner
}

\begin{abstract}
We approach index coding as a special case of rate-distortion with multiple receivers, each with some side information about the source. Specifically, using techniques developed for the rate-distortion problem, we provide two upper bounds and one lower bound on the optimal index coding rate. The upper bounds involve specific choices of the auxiliary random variables in the best existing scheme for the rate-distortion problem. The lower bound is based on a new lower bound for the general rate-distortion problem. The bounds are shown to coincide for a number of (groupcast) index coding instances, including all instances for which the number of decoders does not exceed three.
\end{abstract}

\section{INTRODUCTION}

We consider a general version of the index coding problem, in which a single encoder observes a vector-valued source, the components of which are i.i.d., uniformly-distributed, binary random variables. There are several decoders, each of which has a subset of the source components as side information, and seeks to losslessly reproduce a disjoint subset of source components. The encoder must broadcast a single message to all of the decoders, which allows all of them to reproduce their desired source components. This problem models media distribution over a rate-constrained downlink in which some of the clients already have some of the media to be distributed, which can happen if they cached prior transmissions from the encoder. We seek to understand what rate is required of the encoder's message when the encoder may code over many i.i.d. realizations of the source vector. Specifically, we seek estimates of the minimal rate that are both efficiently computable and provably close to the true minimal rate, at least under some conditions.

The index coding problem has attracted considerable attention since it was introduced (e.g., [1], [2], [3], [4], [5], [6], [7], [8]), and the formulations studied vary along at least three different axis. First, one can impose structure on the demands and the side information. Birk and Kol's paper [1] introducing the problem focused on the case in which each source bit is demanded by exactly one decoder, and if Decoder $i$ has Decoder $j$ 's demand as side information then Decoder $j$ must have Decoder $i$ 's demand as side information. The problem instance can then be represented as an undirected graph, with the nodes representing the source bits (or equivalently, the decoders) and the edges representing the side information pattern. Slightly more generally, one can relax the symmetry assumption to obtain a directed, instead of undirected, graph. Shanmugam et al. [4] call this unicast index coding. We shall consider here the more general version in which each decoder can demand any number of source components and the demands may be overlapping among the decoders. Shanmugam et al. call this groupcast index coding; we shall simply call it index coding.

A second independent axis along which index coding formulations vary is whether one allows for coding over time (vector codes) or whether the code must operate on each time instant separately (scalar codes). We shall focus on the former here, due to its intrinsic importance and its connection with rate-distortion theory. Third, and finally, some works require the decoders to reproduce their demands with zero error [2], [4], [5], [6], [9] while others require that the block-error probability vanish [7], [8]. Yet another possibility is to require that the bit-error probability vanish. This paper shall focus on the latter two.

Irrespective of the formulation, most work on index coding views the problem graph-theoretically [3], [4], [9], as in Birk and Kol's original paper. One can then lower and upper bound the optimal rate using graph-theoretic quantities such as the independence number, the clique-cover number, fractional clique-cover number (e.g. [5]), the min-rank [2] and others [3], [4], [9]. This approach has proven to be successful for showing the utility of coding over blocks for this problem [10], and for showing the utility of nonlinear codes [11]. Many of these graph-theoretic quantities are known to be NP-hard to compute, however, and for the others there is no apparent polynomial-time algorithm. Thus these bounds are only useful theoretically or when numerically solving small examples. A noteworthy exception is Theorem 2 of Blasiak et al., which provides a polynomial-time-computable bound that is within a nontrivial factor of the optimal rate for arbitrary instances. The factor in question is quite large, however.

We approach index coding as a special case of the problem of lossy compression with a single encoder and multiple decoders, each with side information. This more general problem was introduced by Heegard-Berger [12] (but see Kaspi [13]) and is sometimes referred to as the Heegard-Berger problem. Index coding can be viewed as the special case in which the source, at each time, is a vector of i.i.d. uniform bits, the side information of each decoder consists of a subset of the source bits, and the distortion measure for Decoder $i$ is the Hamming distortion between the subset of the source bits that Decoder $i$ seeks to reproduce and Decoder $i$ 's reproduction of that subset. We then consider the minimum rate possible so that all of the decoders can achieve zero distortion.

S. Unal and A. B. Wagner are with Cornell University, School of Electrical \& Computer Engineering, Ithaca, NY 14853 USA (e-mail: su62@cornell.edu, wagner@ece.cornell.edu). This paper was presented in part at the IEEE Int. Symposium on Information Theory (ISIT), Istanbul, July 2013 and Information Theory and Applications Workshop (ITA), San Diego, February 2014. 
Viewing the problem in this way allows us to apply tools from network information theory, such as random coding techniques, binning, the use of auxiliary random variables, etc. Using this approach, we prove two achievable bounds and an impossibility (or "converse") bound. Both of the achievable bounds are built upon the best known achievable bound for the Heegard-Berger problem, which is due to Timo et al. [14]. The Timo et al. scheme involves an optimization over the joint distribution of a large number of auxiliary random variables; we provide two methods for selecting this distribution, the first of which is polynomialtime computable but only yields integer rate bounds, while the second is more complex but can yield fractional rates. The achievability results in this paper are thus unusual in that the emphasis is on algorithms for selecting the joint distribution of auxiliary random variables rather than proving new coding theorems per se. It is worth noting that the Timo et al. result is representative of many achievability results in network information theory that take the form of optimization problems over the joint distribution of auxiliary random variables (e.g., [15]). The task of solving these optimization problems has received little attention in the literature 1

Our impossibility result is related to the "degraded-same-marginals" (DSM) impossibility result for broadcast channels [17], [18]. The idea is that the optimal rate can be computed exactly when the source $S$ and the side information variables $Y_{1}, \ldots, Y_{m}$ can be coupled in such a way that

$$
S \leftrightarrow Y_{\sigma(1)} \leftrightarrow Y_{\sigma(2)} \leftrightarrow \cdots \leftrightarrow Y_{\sigma(m)},
$$

meaning that the random variables form a Markov chain in this order, where $\sigma(\cdot)$ is an arbitrary permutation [14]. We call such an instance one with degraded side information. One may then lower bound a given problem by providing (for example), $Y_{1}$ to Decoder 2, $Y_{1}$ and $Y_{2}$ to Decoder 3, etc., to form a degraded instance whose optimal rate is only lower than that of the original problem. We provide a lower bound in this spirit for the general Heegard-Berger problem that improves somewhat on that obtained via a direct application of the above technique. As such, we shall call it the DSM+ lower bound. When applied to the index coding problem, the DSM+ bound provides the same conclusion as a lower bound due to Blasiak et al., although under slightly weaker hypotheses.

We use the DSM+ lower bound to show that our low-complexity achievable bound equals the optimal rate for any number of source components, so long as the number of decoders does not exceed three ${ }^{2}$ In fact, we show the more general result that the achievable bound equals the optimal rate for any number of source components and any number of decoders so long as each source component is present as side information at all of the decoders, none of the decoders, all but one of the decoders, or all but two of the decoders. It is apparent that every problem with three or fewer decoders must be of this form. We also show that the achievable bound coincides with the optimal rate when none of the source components are "excess," a concept that plays an important role in our achievable scheme and that shall be defined later. Our low-complexity achievable scheme bears some resemblance to the partition multicast scheme of Tehrani, Dimakis, and Neely [6]. Although our scheme does not subsume partition multicast (which is NP-hard to compute [6]), we do show that it is optimal in all explicit instances of the problem for which Tehrani, Dimakis, and Neely show that partition multicast is optimal 3

Although the paper is focused mainly on index coding, the results herein also have some significance for the Heegard-Berger problem. The DSM+ bound, mentioned earlier, is the best general lower bound for this problem, and our conclusive results for the index problem represent some of the few nondegraded instances of the Heegard-Berger problem for which the optimal rate is known (see [19], [20], [21], [22], [23], [24] others). This paper is also the first work that considers algorithms for selecting the distribution of the auxiliary random variables in the Timo et al. scheme.

As noted earlier, this work differs from much of the literature on index coding by approaching the problem as one of rate-distortion, or source coding. Some recent works have also approached the problem as one of channel coding [7], [8], and in particular, interference alignment. One of the advantages of the source coding approach espoused here is that it can readily accommodate richer source models and distortion constraints, including sources with memory, lossy reconstruction of analog sources, etc. The very formulation of index coding presumes that the sources have already been compressed down to i.i.d. uniform bits. Thus the index coding is "separated" from the underlying compression, when in fact there might be some advantage to combining the two, a topic that we shall consider in subsequent work.

This paper is outlined as follows. Section III formulates the Heegard-Berger problem and Section III provides the DSM+ lower bound for it. Section [V] formulates the index coding problem. Section $\mathrm{V}]$ and $\mathrm{VI}$ provide a lower bound and an upper bound for the problem respectively. Section VII describes our first scheme of index coding, and Section VIII provides several optimality results for this scheme, including our results for three decoders. Section IX describes our second scheme.

\section{PRoblem DEFinition}

We begin by considering the general form of the Heegard-Berger problem, as opposed to the index-coding problem in particular. There is a single encoder with source $S$ and there are $m$ decoders. Decoder $i$ has a side information $Y_{i}$ that in general depends on $S$. The encoder sends a message at rate $R$ to the decoders, and Decoder $i$ wishes to reconstruct the source

\footnotetext{
${ }^{1}$ Indeed, the prospect that some of these "single-letter" optimization problems might be intrinsically hard to compute is intriguing and seemingly unexplored (though see Arikan [16]).

${ }^{2}$ Recall that we allow each decoder to demand more than one source component and each source component to be demanded by more than one decoder.

${ }^{3}$ Tehrani et al. also show that partition multicast is optimal for the implicitly-defined class of instances for which clique cover is optimal.
} 
with a given distortion constraint $D_{i}$. The objective is to find the rate distortion tradeoff for this problem setup. This is made precise via the following definitions.

Definition 1. An $\left(n, M, D_{1}, \ldots, D_{m}\right)$ code consists of mappings

$$
\begin{gathered}
f: \mathcal{S}^{n} \rightarrow\{1, \ldots, M\} \\
g_{1}:\{1, \ldots, M\} \times \mathcal{Y}_{1}^{n} \rightarrow \hat{\mathcal{S}}_{1}^{n} \\
g_{2}:\{1, \ldots, M\} \times \mathcal{Y}_{2}^{n} \rightarrow \hat{\mathcal{S}}_{2}^{n} \\
\vdots \\
g_{m}:\{1, \ldots, M\} \times \mathcal{Y}_{m}^{n} \rightarrow \hat{\mathcal{S}}_{m}^{n}, \\
\mathbb{E}\left[\frac{1}{n} \sum_{k=1}^{n} d\left(S_{k}, \hat{S}_{(i) k}\right)\right] \leq D_{i}, \forall i \in[m]
\end{gathered}
$$

where $\mathcal{S}$ denotes the source alphabet, $\mathcal{Y}_{1}, \ldots, \mathcal{Y}_{m}$ denote the side information alphabets at Decoder 1 through $m$ and $\hat{\mathcal{S}}_{1}^{n}, \ldots, \hat{\mathcal{S}}_{m}^{n}$ denote the reconstruction alphabets at Decoder 1 through $m$ and $d(.,.) \in[0 \infty)$ denotes a distortion measure and $[m]=\{1, \ldots, m\}$. Lastly, we call $f$ the encoding function at the encoder and $g_{i}$ the decoding function at Decoder $i$ where $i \in[m]$.

Definition 2. A rate distortion pair $(R, D)$, where $D=\left(D_{1}, \ldots, D_{m}\right)$ is achievable if for every $\epsilon>0$, there exists an $\left(n, M, D_{1}+\epsilon, \ldots, D_{m}+\epsilon\right)$ code such that $n^{-1} \log M \leq R+\epsilon$.

Definition 3. The rate distortion function $R(D)$ is defined as

$$
R(D)=\inf \{R \mid(R, D) \text { is achievable }\}
$$

Finding a computable characterization $R(D)$ is a long-standing open problem in network information theory. Currently, such a characterization is only available for a few special cases. Heegard and Berger themselves [12] provided one when the side information at the decoders is degraded. Watanabe [19] provided one for the case that the source consists of two independent components, the distortion constraints for both decoders are decoupled across the two components, and the side information at the two decoders is degraded "in mismatched order" (see [19] for the precise setup). Sgarro's result [22] implies a characterization for the problem in which two decoders both wish to reproduce the source losslessly, without any assumption on their side information (see also [23]). Timo et al. [24] provide a characterization for the two-decoder case when one decoder's side information is "conditionally less noisy" than the other's and the weaker decoder seeks to losslessly reproduce a deterministic function of the source. Timo et al. [20] solve various two-decoder cases in which the source consists of two components, say $(X, Y)$, and one decoder has $X$ as side information and wants to reconstruct $Y$ while the other has $Y$ as side information and wants to reconstruct $X$. The present authors determined the rate distortion region for the two-decoder problem with vector Gaussian sources and side information, subject to a constraint on the error covariance matrices at the two decoders [21]. Several (nondegraded) special cases in which both decoders wish to losslessly reproduce a function of the source have been solved by Laich and Wigger [25]. Of course, several instances of index coding that are not degraded have also been solved.

A general achievable result, i.e., an upper bound on $R(D)$, was provided by Heegard and Berger [12], which was corrected and extended by Timo et al. [14]. We provide a computable lower bound on $R(D)$ for general instances of the problem in this section. This lower bound will be used later in the paper to solve several index coding instances.

\section{LOWER BOUND FOR A RATE DISTORTION FUNCTION}

We start our analysis by providing a lower bound to the general rate distortion problem.

Theorem 1 (DSM+ Lower Bound). Let the pmf's $P\left(S, Y_{i}\right)$ for all $i \in[m]$ be given. $R(D)$ is lower bounded by

$$
R_{D S M+}(D)=\max _{\sigma} \sup _{\bar{P}} \bar{R}_{\sigma}(D)
$$

where

$$
\begin{gathered}
\bar{R}_{\sigma}(D)=\min _{U_{1}, \ldots, U_{m}}\left[I\left(S ; U_{\sigma(1)} \mid Y_{\sigma(1)}\right)+I\left(S ; U_{\sigma(2)} \mid U_{\sigma(1)}, Y_{\sigma(1)}, Y_{\sigma(2)}\right)+\cdots\right. \\
\left.+I\left(S ; U_{\sigma(m)} \mid U_{\sigma(1)}, \ldots, U_{\sigma(m-1)}, Y_{\sigma(1)}, \ldots, Y_{\sigma(m)}\right)\right]
\end{gathered}
$$

and

1) $\sigma($.$) denotes a permutation on integers [m]$ 
2) $\bar{P}=\left\{P\left(S, Y_{1}, \ldots, Y_{m}\right) \mid \sum_{Y_{j}: j \neq i} P\left(S, Y_{1}, \ldots, Y_{m}\right)=P\left(S, Y_{i}\right), \forall i \in[m]\right\}$

3) $\left(U_{1}, \ldots, U_{m}\right)$ is jointly distributed with $S, Y_{1}, \ldots, Y_{m}$ such that

$$
\left(Y_{1}, \ldots, Y_{m}\right) \leftrightarrow S \leftrightarrow\left(U_{1}, \ldots, U_{m}\right)
$$

and

4) there exist functions $g_{1}, \ldots, g_{m}$ such that

$$
\mathbb{E}\left[d\left(S, g_{\sigma(i)}\left(U_{\sigma(i)}, Y_{\sigma(i)}\right)\right)\right] \leq D_{\sigma(i)} \forall i \in[m],
$$

5) $\left|U_{\sigma(i)}\right| \leq|\mathcal{S}| \prod_{j=1}^{i-1}\left|U_{\sigma(j)}\right|+(m+2-i)$ for all $i \in[m]$.

The idea behind the proof was described in the introduction. Note that since the optimal rate only depends on the source and side information through the "marginals"

$$
\left(S, Y_{i}\right) \quad i \in[m]
$$

we may couple the $Y_{i}$ variables to form a joint distribution $\bar{P}\left(S, Y_{1}, \ldots, Y_{m}\right)$ as we please, leading to the inner optimization in (1). Also note that a direct application of the DSM idea would yield the weaker bound in which $\cup_{j \leq i} Y_{\sigma(j)}$ appears as an argument to $g_{\sigma(i)}$ in 3 .

Proof of Theorem 1 . Let $P\left(S, Y_{i}\right)$ for all $i \in[m]$ be given and let permutation $\sigma(i)=i$ for all $i \in[m]$. Let $(R, D)$ be an achievable rate distortion pair and $\epsilon>0$. Then there exists a $\left(n, M, D_{1}+\epsilon, \ldots, D_{m}+\epsilon\right)$ code for some $n$ such that $\log M \leq n(R+\epsilon)$.

We can write,

$$
\begin{aligned}
n(R+\epsilon) & \geq H(J) \\
& \geq I\left(S^{n}, Y_{1}^{n}, \ldots, Y_{m}^{n} ; J\right)
\end{aligned}
$$

where $J$ is the output of the encoder, $Y_{1}^{n}=\left(Y_{11}, \ldots, Y_{1 n}\right)$ (for the ease of notation we drop the parentheses around the index of the random variable unless it causes ambiguity) and $Y_{11}$ denotes all $Y_{1}^{n}$ but $Y_{1 i}$. Then if we apply the chain rule to $I\left(S^{n}, Y_{1}^{n}, \ldots, Y_{m}^{n} ; J\right)$, right hand side of (4) equals

$$
\begin{aligned}
& I\left(Y_{1}^{n} ; J\right)+I\left(Y_{2}^{n} ; J \mid Y_{1}^{n}\right)+\cdots+I\left(Y_{m}^{n} ; J \mid Y_{1}^{n}, \ldots, Y_{m-1}^{n}\right)+I\left(S^{n} ; J \mid Y_{1}^{n}, \ldots, Y_{m}^{n}\right) \\
& \geq I\left(Y_{2}^{n} ; J \mid Y_{1}^{n}\right)+\cdots+I\left(Y_{m}^{n} ; J \mid Y_{1}^{n}, \ldots, Y_{m-1}^{n}\right)+I\left(S^{n} ; J \mid Y_{1}^{n}, \ldots, Y_{m}^{n}\right) \\
& \geq \sum_{i=1}^{n}\left[I\left(Y_{2 i} ; J, Y_{1 \dot{\imath}} \mid Y_{1 i}\right)+\cdots+I\left(Y_{m i} ; J, Y_{1 \dot{\alpha}}, \ldots, Y_{(m-1) \dot{l}} \mid Y_{1 i}, \ldots, Y_{(m-1) i}\right)+I\left(S_{i} ; J, Y_{1 \dot{1}}, \ldots, Y_{m i \mathfrak{l}} \mid Y_{1 i}, \ldots, Y_{m i}\right)\right] \\
& \stackrel{b}{=} \sum_{i=1}^{n}\left[I\left(Y_{2 i} ; J, Y_{1 \dot{\imath}} \mid Y_{1 i}\right)+\cdots+I\left(Y_{m i} ; J, Y_{1 \dot{1}}, \ldots, Y_{(m-1) \dot{\imath}} \mid Y_{1 i}, \ldots, Y_{(m-1) i}\right)+I\left(S_{i} ; J, Y_{1 \dot{u}}, \ldots, Y_{(m-1) \dot{\imath}} \mid Y_{1 i}, \ldots, Y_{m i}\right)\right. \\
& \left.+I\left(S_{i} ; Y_{m i \mathfrak{l}} \mid J, Y_{1 \ddot{\imath}}, \ldots, Y_{(m-1) \dot{l}}, Y_{1 i}, \ldots, Y_{m i}\right)\right]
\end{aligned}
$$

where $a$ is obtained by the chain rule and $b$ is due to the chain rule applied to the last term. When we combine the second-to-last and third-to-last term above, we get

$$
\begin{aligned}
& n(R+\epsilon) \\
& \geq \sum_{i=1}^{n}\left[I\left(Y_{2 i} ; J, Y_{1 \ddot{1}} \mid Y_{1 i}\right)+\cdots+I\left(Y_{(m-1) i} ; J, Y_{1 \ddot{1}}, \ldots, Y_{(m-2) \dot{\ddot{d}}} \mid Y_{1 i}, \ldots, Y_{(m-2) i}\right)\right.
\end{aligned}
$$

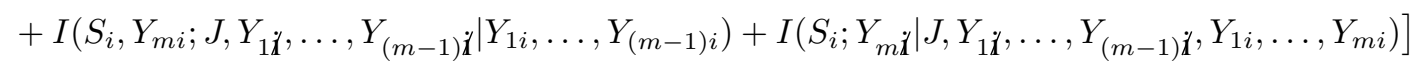

$$
\begin{aligned}
& \geq \sum_{i=1}^{n}\left[I\left(Y_{2 i} ; J, Y_{1 \dot{\imath}} \mid Y_{1 i}\right)+\cdots+I\left(Y_{(m-1) i} ; J, Y_{1 \dot{1}}, \ldots, Y_{(m-2) \dot{\mathfrak{l}}} \mid Y_{1 i}, \ldots, Y_{(m-2) i}\right)\right. \\
& \left.+I\left(S_{i} ; J, Y_{1 \ddot{x}}, \ldots, Y_{(m-1) \dot{\imath}} \mid Y_{1 i}, \ldots, Y_{(m-1) i}\right)+I\left(S_{i} ; Y_{m \ddot{l}} \mid J, Y_{1 \ddot{x}}, \ldots, Y_{(m-1) \dot{l}}, Y_{1 i}, \ldots, Y_{m i}\right)\right] .
\end{aligned}
$$

Now we apply the chain rule on the second-to-last term, giving

$$
\begin{aligned}
& n(R+\epsilon) \\
& \geq \sum_{i=1}^{n}\left[I\left(Y_{2 i} ; J, Y_{1 \mathfrak{i}} \mid Y_{1 i}\right)+\cdots+I\left(Y_{(m-1) i} ; J, Y_{1 \dot{1}}, \ldots, Y_{(m-2) \dot{\imath}} \mid Y_{1 i}, \ldots, Y_{(m-2) i}\right)\right. \\
& +I\left(S_{i} ; J, Y_{1 \ddot{u}}, \ldots, Y_{(m-2) \dot{\imath}} \mid Y_{1 i}, \ldots, Y_{(m-1) i}\right)+I\left(S_{i} ; Y_{(m-1) \dot{\imath}} \mid J, Y_{1 \ddot{l}}, \ldots, Y_{(m-2) \dot{l}}, Y_{1 i}, \ldots, Y_{(m-1) i}\right) \\
& \left.+I\left(S_{i} ; Y_{m i \mathfrak{i}} \mid J, Y_{1 \ddot{1}}, \ldots, Y_{(m-1) \dot{l}}, Y_{1 i}, \ldots, Y_{m i}\right)\right]
\end{aligned}
$$




$$
\begin{aligned}
& \stackrel{c}{=} \sum_{i=1}^{n}\left[I\left(Y_{2 i} ; J, Y_{1 \dot{\imath}} \mid Y_{1 i}\right)+\cdots+I\left(Y_{(m-2) i} ; J, Y_{1 \dot{1}}, \ldots, Y_{(m-3) \dot{\imath}} \mid Y_{1 i}, \ldots, Y_{(m-3) i}\right)\right. \\
& +I\left(S_{i}, Y_{(m-1) i} ; J, Y_{1 \dot{\alpha}}, \ldots, Y_{(m-2) \dot{\mid}} \mid Y_{1 i}, \ldots, Y_{(m-2) i}\right)+I\left(S_{i} ; Y_{(m-1) \dot{l}} \mid J, Y_{1 \dot{\alpha}}, \ldots, Y_{(m-2) \dot{l}}, Y_{1 i}, \ldots, Y_{(m-1) i}\right) \\
& \left.+I\left(S_{i} ; Y_{m i \mathfrak{l}} \mid J, Y_{1 \ddot{1}}, \ldots, Y_{(m-1) \dot{l}}, Y_{1 i}, \ldots, Y_{m i}\right)\right] \\
& \geq \sum_{i=1}^{n}\left[I\left(Y_{2 i} ; J, Y_{1 \dot{\imath}} \mid Y_{1 i}\right)+\cdots+I\left(Y_{(m-2) i} ; J, Y_{1 \ddot{u}}, \ldots, Y_{(m-3) \dot{\imath}} \mid Y_{1 i}, \ldots, Y_{(m-3) i}\right)\right. \\
& +I\left(S_{i} ; J, Y_{1 \dot{\imath}}, \ldots, Y_{(m-2) \dot{\imath}} \mid Y_{1 i}, \ldots, Y_{(m-2) i}\right)+I\left(S_{i} ; Y_{(m-1) \dot{\imath}} \mid J, Y_{1 \ddot{l}}, \ldots, Y_{(m-2) \dot{l}}, Y_{1 i}, \ldots, Y_{(m-1) i}\right) \\
& \left.+I\left(S_{i} ; Y_{m \ddot{1}} \mid J, Y_{1 \ddot{1}}, \ldots, Y_{(m-1) \dot{l}}, Y_{1 i}, \ldots, Y_{m i}\right)\right]
\end{aligned}
$$

where, $c$ is obtained by combining third-to-last and fourth-to-last terms in (8). Note that (7) is obtained from (6) by applying a series of chain rules and term combinations. If we continue this procedure as we did while obtaining (9) from (7), we get

$$
\begin{aligned}
& R+\epsilon \\
& \geq \frac{1}{n} \sum_{i=1}^{n}\left[I\left(S_{i} ; J, Y_{1 \dot{\imath}} \mid Y_{1 i}\right)+I\left(S_{i} ; Y_{2 \dot{\imath}} \mid J, Y_{1 \dot{1}}, Y_{1 i}, Y_{2 i}\right)+\cdots+I\left(S_{i} ; Y_{m \dot{\imath}} \mid J, Y_{1 \dot{\imath}}, \ldots, Y_{(m-1) \dot{\mathbf{l}}}, Y_{1 i}, \ldots, Y_{m i}\right)\right] \\
& \stackrel{a}{=} \frac{1}{n} \sum_{i=1}^{n}\left[I\left(S_{i} ; U_{1 i} \mid Y_{1 i}\right)+I\left(S_{i} ; U_{2 i} \mid U_{1 i}, Y_{1 i}, Y_{2 i}\right)+\cdots+I\left(S_{i} ; U_{m i} \mid U_{1 i}, \ldots, U_{(m-1) i}, Y_{1 i}, \ldots, Y_{m i}\right)\right] \\
& \stackrel{b}{=} \frac{1}{n} \sum_{i=1}^{n}\left[I\left(S_{i} ; U_{1 i} \mid Y_{1 i}, T=i\right)+I\left(S_{i} ; U_{2 i} \mid U_{1 i}, Y_{1 i}, Y_{2 i}, T=i\right)+\cdots+I\left(S_{i} ; U_{m i} \mid U_{1 i}, \ldots, U_{(m-1) i}, Y_{1 i}, \ldots, Y_{m i}, T=i\right)\right] \\
& \stackrel{c}{=} I\left(S ; U_{1} \mid Y_{1}\right)+I\left(S ; U_{2} \mid U_{1}, Y_{1}, Y_{2}\right)+\cdots+I\left(S ; U_{m} \mid U_{1}, \ldots, U_{(m-1)}, Y_{1}, \ldots, Y_{m}\right)
\end{aligned}
$$

where,

$\mathrm{a}: U_{j i}=\left(J, Y_{j i}\right), \forall j \in[m]$

$\mathrm{b}: T$ is a random variable uniformly distributed on $[\mathrm{m}]$ and independent of all source and side information variables and the $U_{j i} \mathrm{~s}$.

c: Relabel $\left(U_{i}, T\right)$ as $U_{i}$ for all $i \in[m]$.

Note that $\left(U_{1}, \ldots, U_{m}\right)$ satisfies conditions 3$)$ and 4). By Lemma 4 in the Appendix, we can obtain the cardinality bounds on $\left(U_{1}, \ldots, U_{m}\right)$ as in condition 5). Then we minimize the right hand side of (11) over $\left(U_{1}, \ldots, U_{m}\right)$. Since the problem can be described by specifying only the marginal $P\left(S, Y_{i}\right)$ 's, we optimize it over the set of joint distributions such that the marginal $P\left(S, Y_{i}\right)$ 's are the same. This gives us a lower bound to $R(D)$. Lastly note that, we fixed the permutation as $\sigma(i)=i$ for all $i \in[m]$ and to get (5), we applied the chain rule to (4) in the following order. We started with $Y_{1}^{n}$ then continued with $Y_{2}^{n}, \ldots, Y_{m}^{n}$ and lastly we had $S^{n}$. Since we have $m$ decoders with side information, we can get $m$ ! different permutations. Hence, applying a similar procedure to all permutations, we get $m$ ! lower bounds. By taking their maximum, we obtain a lower bound, $R_{D S M+}(D+\epsilon \mathbf{1})$, where 1 denotes the $m \times 1$ vector with all components 1 .

Hence we have,

$$
R(D) \geq R_{D S M+}(D+\epsilon \mathbf{1})-\epsilon .
$$

Lemma 1. $R_{D S M+}(D+\epsilon \mathbf{1})$ of Theorem 1 is continuous in $\epsilon$ from the right at $\epsilon=0$.

Proof of Lemma 가. Since finite maximum of functions that are continuous from the right is also continuous from the right, it is enough to prove that $\sup _{\bar{P}} \bar{R}_{\sigma}(D+\epsilon \mathbf{1})$ is continuous in $\epsilon$ from right.

First we show that for a given joint distribution $P\left(S, Y_{1}, \ldots, Y_{m}\right), \bar{R}_{\sigma}(D+\epsilon \mathbf{1})$ is continuous in $\epsilon$ from right for a given permutation $\sigma($.$) .$

Let $\epsilon_{k}$ be a monotonically decreasing sequence converging to 0 and let $U_{1}\left(D+\epsilon_{k} \mathbf{1}\right), \ldots, U_{m}\left(D+\epsilon_{k} \mathbf{1}\right)$ denote an optimal $\left(U_{1}, \ldots, U_{m}\right)$ which gives $\bar{R}_{\sigma}\left(D+\epsilon_{k} \mathbf{1}\right)$. Since the cardinalities of $\left(U_{1}, \ldots, U_{m}\right)$ are finite, together with the conditions 3$)$ and 4), we have an optimization over a compact set. Then, we can find a convergent subsequence $\epsilon_{s_{k}}$ such that $U_{1}(D+$ $\left.\epsilon_{s_{k}} \mathbf{1}\right), \ldots, U_{m}\left(D+\epsilon_{s_{k}} \mathbf{1}\right)$ converges to a $\left(U_{1}, \ldots, U_{m}\right)$ which is feasible for the distortion $D$. Hence we have

$$
\liminf _{\epsilon \rightarrow 0} \bar{R}_{\sigma}(D+\epsilon \mathbf{1}) \geq \bar{R}_{\sigma}(D) .
$$

Also, since $\bar{R}_{\sigma}(D)$ is non increasing function of $D$ we can write

$$
\limsup _{\epsilon \rightarrow 0} \bar{R}_{\sigma}(D+\epsilon \mathbf{1}) \leq \bar{R}_{\sigma}(D),
$$


concluding that $\bar{R}_{\sigma}(D+\epsilon \mathbf{1})$ is continuous in $\epsilon$ from the right for a given permutation $\sigma($.$) .$

Now we show that $\sup _{\bar{P}} \bar{R}_{\sigma}(D+\epsilon \mathbf{1})$ is continuous in $\epsilon$ from the right. Let us temporarily write $\bar{R}_{\sigma}(D)$ as $\bar{R}_{\sigma}(\bar{P}, D)$ to indicate the dependence on $\bar{P}$. Then for any $\epsilon>0$ and any $\bar{P}$, we have $\bar{R}_{\sigma}(\bar{P}, D+\epsilon \mathbf{1}) \leq \bar{R}_{\sigma}(\bar{P}, D)$. Hence we have

$$
\limsup _{\epsilon \rightarrow 0} \sup _{\bar{P}} \bar{R}_{\sigma}(\bar{P}, D+\epsilon \mathbf{1}) \leq \sup _{\bar{P}} \bar{R}_{\sigma}(\bar{P}, D) .
$$

Now, we fix any $\delta>0$. Then there exists $P^{\prime}$ such that $\bar{R}_{\sigma}\left(P^{\prime}, D\right) \geq \sup _{\bar{P}} \bar{R}_{\sigma}(\bar{P}, D)-\frac{\delta}{2}$. Let $\epsilon>0$ be such that $\bar{R}_{\sigma}\left(P^{\prime}, D+\epsilon \mathbf{1}\right) \geq \bar{R}_{\sigma}\left(P^{\prime}, D\right)-\frac{\delta}{2}$. Then for any $0<\epsilon^{\prime}<\epsilon$ we can write

$$
\begin{aligned}
\sup _{\bar{P}} \bar{R}_{\sigma}(\bar{P}, D) & \leq \bar{R}_{\sigma}\left(P^{\prime}, D\right)+\frac{\delta}{2} \\
& \leq \bar{R}_{\sigma}\left(P^{\prime}, D+\epsilon^{\prime} \mathbf{1}\right)+\delta \\
& \leq \sup _{\bar{P}} \bar{R}_{\sigma}\left(\bar{P}, D+\epsilon^{\prime} \mathbf{1}\right)+\delta,
\end{aligned}
$$

implying that

$$
\sup _{\bar{P}} \bar{R}_{\sigma}(\bar{P}, D)-\delta \leq \liminf _{\epsilon^{\prime} \rightarrow 0} \sup _{\bar{P}} \bar{R}_{\sigma}\left(\bar{P}, D+\epsilon^{\prime} \mathbf{1}\right) .
$$

Since $\delta>0$ was arbitrary, (13) and 14) give the result.

By Lemma 1, when $\epsilon$ goes to 0 , 12) becomes

$$
R(D) \geq R_{D S M+}(D)
$$

Remark 1. Since the proof constructs the $U_{i}$ variables in a way that does not depend on the permutation $\sigma(\cdot)$ or the coupling $\bar{P}$, one could state the bound with the minimum over $U_{1}, \ldots, U_{m}$ outside the maximum over $\sigma(\cdot)$ and $\bar{P}$. This complicates the proof of the cardinality bounds in 5), however, and the maxmin form of the bound is sufficient for the purposes of this paper, so we shall defer consideration of this potential strengthening to a later work.

Next, we turn our focus to the index coding problem which can be viewed as a special case of the Heegard-Berger problem.

\section{INDEX CODING : PROBLEM Formulation}

For the $m$ user index coding problem, each decoder $\alpha$ wants to reconstruct $\mathbf{f}_{\alpha}$, which is an arbitrary subset of the source $\mathbf{S}$, that is, a collection of i.i.d. Bernoulli $\left(\frac{1}{2}\right)$ bits at the encoder. There may be overlapping demands, i.e., more than one decoder may demand the same bit. Also, each Decoder $\alpha$ has side information $\mathbf{Y}_{\alpha}$ consisting of an arbitrary subset of the source. We assume that decoders do not demand a component of their own side information since they already have it, and we assume that $\mathbf{Y}_{\alpha} \neq \mathbf{Y}_{\beta}$, for all $\alpha \neq \beta$ since we can combine two decoders if they have the same side information. We may also assume that every source bit is demanded by at least one decoder, for otherwise that bit may be completely purged from the system.

Let $\mathbf{S}_{\mathbf{J}}$ denote the part of the source which each decoder in a subset $J$ of $[m]$ does not have and all decoders in $[m] \backslash J$ have as side information. If $J=\{\alpha\}$, i.e., a singleton, then for ease of notation we use $\mathbf{S}_{\alpha}$ instead of $\mathbf{S}_{\{\alpha\}}$. Since there are $m$ decoders, we group the elements of $\mathbf{S}$ into $2^{m}$ disjoint sets such that $\mathbf{S}=\cup_{J \subseteq[m]} \mathbf{S}_{\mathbf{J}}$. Note that each $\mathbf{S}_{\mathbf{J}}$ may be empty, may consist of a single bit, or may consist of multiple bits.

Let $G_{0}=\mathbf{S}_{[\mathbf{m}]}$ denote the elements of the source that none of the decoders have, $G_{m}=\mathbf{S}_{\emptyset}$ denote the elements all decoders have, $G_{m-1}=\cup_{\alpha \in[m]} \mathbf{S}_{\alpha}$ denote elements that $m-1$ of the decoders have, $G_{m-2}=\cup_{\{\alpha, \beta\} \subseteq[m]} \mathbf{S}_{\{\alpha, \beta\}}$ denote elements that $m-2$ of the decoders have and so on. To ease the notation for the rest of the paper, whenever we write a set $\{\alpha, \beta\}$, we assume $\alpha \neq \beta$ unless otherwise stated. Then $\mathbf{S}$ can be represented as $\mathbf{S}=\left\{G_{0}, G_{m}, G_{m-1}, \ldots, G_{1}\right\}$, as shown in Fig. 1 .

The demand $\mathbf{f}_{\alpha}$ at Decoder $\alpha$ can be written in terms of components $\mathbf{S}_{\mathbf{J}}$ of $\mathbf{S}$. For this, we introduce the following notation.

Let $f_{I J}$ denote the demand that is a subset of source $\mathbf{S}_{\mathbf{J}}$ and is required by each decoder in a subset $I$ of $[m]$ and by no decoders in $[m] \backslash I$. If $I=\{\alpha\}$, then for ease of notation we use $f_{\alpha J}$ instead of $f_{\{\alpha\} J}$. We will generally assume that $I \subseteq J$ since only decoders in $J$ may have a demand about $\mathbf{S}_{\mathbf{J}}$ and decoders in $[m] \backslash J$ already have $\mathbf{S}_{\mathbf{J}}$ as side information. If $I \nsubseteq J$, $f_{I J}$ is empty. Also, $f_{I J}$ and $f_{K J}$ are independent (i.e., $f_{I J} \perp f_{K J}$ ) for all possible choices of $I, K$ and $J$ with $I \neq K$ since $f_{I J} \cap f_{K J}=\emptyset$ unless $I=K$. Lastly, each $f_{I J}$ may be empty, a single bit or may consist of multiple bits.

We have written the source as $\mathbf{S}=\left\{G_{0}, G_{m}, G_{m-1}, \ldots, G_{1}\right\}$ and the demands in terms of $\mathbf{S}_{\mathbf{J}}$ 's. From now on, we consider an ordered set structure on $\mathbf{S}$ which naturally induces orders on $\mathbf{S}_{\mathbf{J}}$ 's. Then each demand $f_{I J}$ is also an ordered set that can also be viewed as a vector. In fact, we shall find it convenient to view $f_{I J}, \mathbf{S}$, and other similar quantities at times as sets and at times as vectors. 


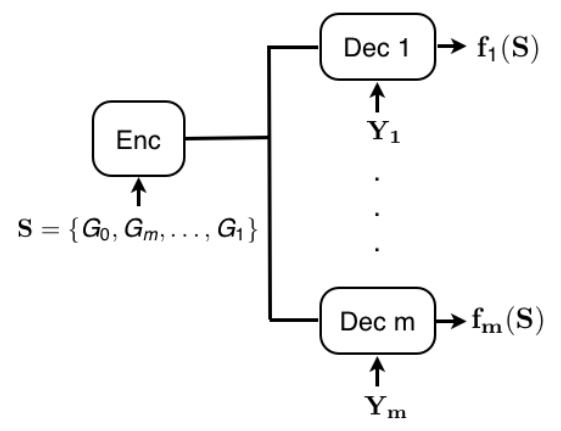

Fig. 1. Index coding with $m$ users.

Since this problem can be considered as a special case of the Heegard-Berger problem, we use a similar definition for the code except for the distortion. Specifically, we consider block error probabilities instead of the distortion constraints stated in Definition 1. Hence, we use the following definitions for the code, error and optimal rate.

Definition 4. Let $\mathcal{S}$ denote the alphabet of $\mathbf{S}$. An $(n, M)$ code consists of mappings

$$
\begin{aligned}
& f: \mathcal{S}^{n} \rightarrow\{1, \ldots, M\} \\
& g_{1}:\{1, \ldots, M\} \times \mathcal{Y}_{1}^{n} \rightarrow \mathcal{F}_{1}^{n} \\
& g_{2}:\{1, \ldots, M\} \times \mathcal{Y}_{2}^{n} \rightarrow \mathcal{F}_{2}^{n} \\
& \vdots \\
& g_{m}:\{1, \ldots, M\} \times \mathcal{Y}_{m}^{n} \rightarrow \mathcal{F}_{m}^{n},
\end{aligned}
$$

where we $f$ denotes the encoding function at the encoder and $g_{\alpha}$ denotes the decoding function at Decoder $\alpha$ where $\alpha \in[m]$.

Definition 5. The probability of error for a given code is defined as

$$
P_{e}=\operatorname{Pr}\left\{g_{1}\left(f\left(\mathbf{S}^{\mathbf{n}}\right), \mathbf{Y}_{\mathbf{1}}^{\mathbf{n}}\right) \neq \mathbf{f}_{\mathbf{1}}^{\mathbf{n}}\left(\mathbf{S}^{\mathbf{n}}\right) \cup g_{2}\left(f\left(\mathbf{S}^{\mathbf{n}}\right), \mathbf{Y}_{\mathbf{2}}^{\mathbf{n}}\right) \neq \mathbf{f}_{\mathbf{2}}^{\mathbf{n}}\left(\mathbf{S}^{\mathbf{n}}\right), \ldots, \cup g_{m}\left(f\left(\mathbf{S}^{\mathbf{n}}\right), \mathbf{Y}_{\mathbf{m}}^{\mathbf{n}}\right) \neq \mathbf{f}_{\mathbf{m}}^{\mathbf{n}}\left(\mathbf{S}^{\mathbf{n}}\right)\right\} .
$$

Then achievability and optimal rate can be defined as follows.

Definition 6. The rate $R$ is achievable if there exists a sequence of $(n, M)$ codes with rate $n^{-1} \log M \leq R$ such that the probability of error, $P_{e}$, tends to zero as $n$ tends to infinity.

Definition 7. The optimal rate $R_{\text {opt }}$ is defined as

$$
R_{o p t}=\inf \{R \mid R \text { is achievable }\}
$$

We shall call the problem defined in this section index coding, although most existing work on index coding requires the code to achieve zero, as opposed to vanishing, block error [4], [5], [6]. In support of the definitions adopted here, see [7], [8] for works that use vanishing block error probability and [26] for results connecting the two formulations.

\section{LOWER BOUND FOR INDEX CODING}

The next theorem gives a lower bound to the index coding problem using the DSM+ lower bound from Section III.

Theorem 2. The optimal rate of the index coding problem is lower bounded by

$$
R_{D S M+}=\max _{\sigma}\left[H\left(\mathbf{f}_{\sigma(\mathbf{1})} \mid \mathbf{Y}_{\sigma(\mathbf{1})}\right)+H\left(\mathbf{f}_{\sigma(\mathbf{2})} \mid \mathbf{f}_{\sigma(\mathbf{1})}, \mathbf{Y}_{\sigma(\mathbf{1})}, \mathbf{Y}_{\sigma(\mathbf{2})}\right)+\cdots+H\left(\mathbf{f}_{\sigma(\mathbf{m})} \mid \mathbf{f}_{\sigma(\mathbf{1})}, \ldots, \mathbf{f}_{\sigma(\mathbf{m}-\mathbf{1})}, \mathbf{Y}_{\sigma(\mathbf{1})}, \ldots, \mathbf{Y}_{\sigma(\mathbf{m})}\right)\right]
$$

where $\sigma($.$) denotes a permutation on integers [m]$.

Proof of Theorem 2 .

We will use the lower bound in Theorem 1 to prove the theorem. Note that this lower bound is for per-letter distortion constraints but it can be adapted to handle block error probabilities in the following way. Vanishing error probability, $P_{e}$, for index coding problem implies vanishing block error probability for each Decoder $i$, i.e., $\operatorname{Pr}\left(g_{i}\left(f\left(\mathbf{S}^{\mathbf{n}}\right), \mathbf{Y}_{\mathbf{i}}^{\mathbf{n}}\right) \neq \mathbf{f}_{\mathbf{i}}^{\mathbf{n}}\left(\mathbf{S}^{\mathbf{n}}\right)\right)$, which 
implies vanishing distortion with respect to Hamming distortion measure for Decoder $i$. Also, note that lower bound in Theorem 1 is continuous from right by Lemma 1 . Hence, the optimal rate for the index coding problem, $R_{o p t}$, is lower bounded by

$$
\begin{aligned}
R_{o p t} \geq \max _{\sigma} \min _{U_{1}, \ldots, U_{m}}\left[I\left(\mathbf{S} ; U_{\sigma(1)} \mid \mathbf{Y}_{\sigma(\mathbf{1})}\right)+I\left(\mathbf{S} ; U_{\sigma(2)} \mid U_{\sigma(1)}, \mathbf{Y}_{\sigma(\mathbf{1})}, \mathbf{Y}_{\sigma(\mathbf{2})}\right)+\cdots\right. \\
\left.+I\left(\mathbf{S} ; U_{\sigma(m)} \mid U_{\sigma(1)}, \ldots, U_{\sigma(m-1)}, \mathbf{Y}_{\sigma(\mathbf{1})}, \ldots, \mathbf{Y}_{\sigma(\mathbf{m})}\right)\right]
\end{aligned}
$$

such that

1) $\sigma($.$) denotes a permutation on integers [\mathrm{m}]$

2) $\left(U_{1}, \ldots, U_{m}\right)$ jointly distributed with $\mathbf{S}, \mathbf{Y}_{\mathbf{1}}, \ldots, \mathbf{Y}_{\mathbf{m}}$, and

$$
H\left(\mathbf{f}_{\sigma(\mathbf{i})} \mid U_{\sigma(i)}, \mathbf{Y}_{\sigma(\mathbf{i})}\right)=0, \forall i \in[m] .
$$

Note that the maximum over $\bar{P}$ in $[1]$ is degenerate for index coding. Without loss of generality let $\sigma(i)=i$ for all $i \in[m]$. Then we have,

$$
R_{o p t} \geq \min _{U_{1}, \ldots, U_{m}}\left[I\left(\mathbf{S} ; U_{1} \mid \mathbf{Y}_{\mathbf{1}}\right)+I\left(\mathbf{S} ; U_{2} \mid U_{1}, \mathbf{Y}_{\mathbf{1}}, \mathbf{Y}_{\mathbf{2}}\right)+\cdots+I\left(\mathbf{S} ; U_{m} \mid U_{1}, \ldots, U_{m-1}, \mathbf{Y}_{\mathbf{1}}, \ldots, \mathbf{Y}_{\mathbf{m}}\right)\right]
$$

To find an explicit expression for [18, we use the following lemma.

Lemma 2. For $j \in[m]$ we define

$$
K_{j}=\sum_{i=1}^{j-1} I\left(\mathbf{f}_{\mathbf{i}} ; U_{1}^{i} \mid \mathbf{Y}_{\mathbf{1}}^{\mathbf{i}}, \mathbf{f}_{\mathbf{1}}^{\mathbf{i}-\mathbf{1}}\right)+I\left(\mathbf{S} ; U_{1}^{j} \mid \mathbf{Y}_{\mathbf{1}}^{\mathbf{j}}, \mathbf{f}_{\mathbf{1}}^{\mathbf{j}-\mathbf{1}}\right)+\sum_{i=j+1}^{m} I\left(\mathbf{S} ; U_{i} \mid \mathbf{Y}_{\mathbf{1}}^{\mathbf{i}}, U_{1}^{i-1}\right),
$$

where $\mathbf{f}_{\mathbf{1}}^{\mathbf{i}}=\left(\mathbf{f}_{\mathbf{1}}, \ldots, \mathbf{f}_{\mathbf{i}}\right)$ and likewise for $U_{1}^{i}$ etc. Then $K_{1} \geq K_{2} \geq \ldots \geq K_{m}$.

Proof of Lemma 2. We fix any $j \in[m-1]$ and write,

$$
\begin{aligned}
& K_{j}-K_{j+1} \\
& =-I\left(\mathbf{f}_{\mathbf{j}} ; U_{1}^{j} \mid \mathbf{Y}_{\mathbf{1}}^{\mathbf{j}}, \mathbf{f}_{\mathbf{1}}^{\mathbf{j}-\mathbf{1}}\right)+I\left(\mathbf{S} ; U_{1}^{j} \mid \mathbf{Y}_{\mathbf{1}}^{\mathbf{j}}, \mathbf{f}_{\mathbf{1}}^{\mathbf{j}-\mathbf{1}}\right)-I\left(\mathbf{S} ; U_{1}^{j+1} \mid \mathbf{Y}_{\mathbf{1}}^{\mathbf{j}+\mathbf{1}}, \mathbf{f}_{\mathbf{1}}^{\mathbf{j}}\right)+I\left(\mathbf{S} ; U_{j+1} \mid \mathbf{Y}_{\mathbf{1}}^{\mathbf{j}+\mathbf{1}}, U_{1}^{j}\right) \\
& \stackrel{a}{=} I\left(\mathbf{S} ; U_{1}^{j} \mid \mathbf{Y}_{\mathbf{1}}^{\mathbf{j}}, \mathbf{f}_{\mathbf{1}}^{\mathbf{j}}\right)-I\left(\mathbf{S} ; U_{1}^{j+1} \mid \mathbf{Y}_{\mathbf{1}}^{\mathbf{j}+\mathbf{1}}, \mathbf{f}_{\mathbf{1}}^{\mathbf{j}}\right)+I\left(\mathbf{S} ; U_{j+1} \mid \mathbf{Y}_{\mathbf{1}}^{\mathbf{j}+\mathbf{1}}, U_{1}^{j}\right) \\
& =I\left(\mathbf{S} ; U_{1}^{j} \mid \mathbf{Y}_{\mathbf{1}}^{\mathbf{j}}, \mathbf{f}_{\mathbf{1}}^{\mathbf{j}}\right)-I\left(\mathbf{S} ; U_{1}^{j} \mid \mathbf{Y}_{\mathbf{1}}^{\mathbf{j}+\mathbf{1}}, \mathbf{f}_{\mathbf{1}}^{\mathbf{j}}\right)-I\left(\mathbf{S} ; U_{j+1} \mid \mathbf{Y}_{\mathbf{1}}^{\mathbf{j}+\mathbf{1}}, \mathbf{f}_{\mathbf{1}}^{\mathbf{j}}, U_{1}^{j}\right)+I\left(\mathbf{S} ; U_{j+1} \mid \mathbf{Y}_{\mathbf{1}}^{\mathbf{j}+\mathbf{1}}, U_{1}^{j}\right) \\
& \stackrel{b}{\geq} 0,
\end{aligned}
$$

where

a: is due to the chain rule.

$\mathrm{b}$ : is due to the side information and reconstructions being subsets of the source, $\mathbf{S}$.

Then (18) becomes,

$$
\begin{aligned}
& R_{o p t} \\
& \geq K_{1} \\
& \geq K_{m}, \text { by Lemma } 2 \\
& =\sum_{i=1}^{m-1} I\left(\mathbf{f}_{\mathbf{i}} ; U_{1}^{i} \mid \mathbf{Y}_{\mathbf{1}}^{\mathbf{i}}, \mathbf{f}_{\mathbf{1}}^{\mathbf{i}-\mathbf{1}}\right)+I\left(\mathbf{S} ; U_{1}^{m} \mid \mathbf{Y}_{\mathbf{1}}^{\mathbf{m}}, \mathbf{f}_{\mathbf{1}}^{\mathbf{m}-\mathbf{1}}\right) \\
& \geq \sum_{i=1}^{m} I\left(\mathbf{f}_{\mathbf{i}} ; U_{1}^{i} \mid \mathbf{Y}_{\mathbf{1}}^{\mathbf{i}}, \mathbf{f}_{\mathbf{1}}^{\mathbf{i}-\mathbf{1}}\right) \\
& =\sum_{i=1}^{m} H\left(\mathbf{f}_{\mathbf{i}} \mid \mathbf{Y}_{\mathbf{1}}^{\mathbf{i}}, \mathbf{f}_{\mathbf{1}}^{\mathbf{i}-\mathbf{1}}\right)-H\left(\mathbf{f}_{\mathbf{i}} \mid U_{1}^{i}, \mathbf{Y}_{\mathbf{1}}^{\mathbf{i}}, \mathbf{f}_{\mathbf{1}}^{\mathbf{i}-\mathbf{1}}\right) \\
& \left.=\sum_{i=1}^{m} H\left(\mathbf{f}_{\mathbf{i}} \mid \mathbf{Y}_{\mathbf{1}}^{\mathbf{i}}, \mathbf{f}_{\mathbf{1}}^{\mathbf{i}-\mathbf{1}}\right), \text { from } 17\right) .
\end{aligned}
$$

Applying the same procedure to all $m$ ! permutations gives the result.

Remark 2. Evidently the proof shows that the conclusion holds even if one only requires that the bit-error probability, as opposed to the block-error probability, vanish. 
Remark 3. Let us consider one of the $m$ ! expressions of the lower bound in Theorem 2, say the one in (19). We can rewrite it as

$$
\begin{aligned}
& H\left(\mathbf{f}_{\mathbf{1}} \backslash \mathbf{Y}_{\mathbf{1}}\right)+H\left(\mathbf{f}_{\mathbf{2}} \backslash\left\{\mathbf{f}_{\mathbf{1}} \cup \mathbf{Y}_{\mathbf{1}} \cup \mathbf{Y}_{\mathbf{2}}\right\}\right)+\cdots+H\left(\mathbf{f}_{\mathbf{m}} \backslash\left\{\mathbf{Y}_{\mathbf{m}}, \cup_{i=1}^{m-1}\left\{\mathbf{Y}_{\mathbf{i}}, \mathbf{f}_{\mathbf{i}}\right\}\right\}\right) \\
& =\left|\mathbf{f}_{\mathbf{1}} \backslash \mathbf{Y}_{\mathbf{1}}\right|+\left|\mathbf{f}_{\mathbf{2}} \backslash\left\{\mathbf{f}_{\mathbf{1}} \cup \mathbf{Y}_{\mathbf{1}} \cup \mathbf{Y}_{\mathbf{2}}\right\}\right|+\cdots+\left|\mathbf{f}_{\mathbf{m}} \backslash\left\{\mathbf{Y}_{\mathbf{m}}, \cup_{i=1}^{m-1}\left\{\mathbf{Y}_{\mathbf{i}}, \mathbf{f}_{\mathbf{i}}\right\}\right\}\right| .
\end{aligned}
$$

Blasiak et al. [5] define an expanding sequence of decoders as one for which each decoder in the sequence demands a bit that is not contained in the union of the demands and the side information of the decoders that appear earlier in the sequence. Blasiak et al. prove that the size of a largest expanding sequence is a lower bound on the optimal rate. Writing the above bound as in (20) shows that it coincides with the Blasiak et al. bound when each decoder demands a single bit. Of course, the more general case in which a decoder may demand multiple bits can be obtained from the Blasiak et al. result by replacing each such decoder with multiple decoders that each demand a single bit. The Blasiak et al. result does not quite imply Theorem 2 . however, since the former assumes a zero-error formulation (though one could appeal to a result of Langberg and Effros [26] to relate the two formulations).

\section{Achievable Scheme For Index Coding}

For our achievable scheme for index coding, we rely on an achievability result of Timo et al. [14], mentioned earlier, for the general Heegard-Berger problem (see also Heegard and Berger [12]). Since the Timo et al. scheme is rather complicated, we shall state it in a substantially weakened form that will be sufficient for our purposes.

Proposition 1. (cf. [14] Theorem 2]) ${ }^{4}$ The optimal rate $R_{\text {opt }}$ of an index coding problem is upper bounded by

$$
\min \sum_{I \subseteq[m]}\left[\max _{i \in I} H\left(U_{I} \mid \mathbf{Y}_{\mathbf{i}}\right)\right]
$$

where the minimization is over the set of all random variables $U_{I}$ jointly distributed with $\mathbf{S}$ such that

1) There exist functions

$g_{1}\left(\cup_{1 \in I} U_{I}, \mathbf{Y}_{\mathbf{1}}\right), \ldots, g_{m}\left(\cup_{m \in I} U_{I}, \mathbf{Y}_{\mathbf{m}}\right)$ such that

$$
g_{i}\left(\cup_{i \in I} U_{I}, \mathbf{Y}_{\mathbf{i}}\right)=\mathbf{f}_{\mathbf{i}}(\mathbf{S}), \text { for all } i \in[m] .
$$

2) The auxiliary random variables $U_{I}, I \subseteq[m]$ are independent, and for all collections of subsets $J_{1}, \ldots J_{j}, K_{1}, \ldots K_{k}$, $L_{1}, \ldots, L_{l}$, and all subsets $\left\{i_{1}, \ldots, i_{p}\right\} \subseteq[m]$, we have that $\left(U_{J_{1}}, \ldots, U_{J_{j}}\right)$ and $\left(U_{K_{1}}, \ldots, U_{K_{k}}\right)$ are conditionally independent given $\left(\left(U_{L_{1}}, \ldots, U_{L_{l}}\right),\left(\mathbf{Y}_{\mathbf{i}_{1}}, \ldots, \mathbf{Y}_{\mathbf{i}_{\mathbf{p}}}\right)\right.$, provided that the collections $J_{1}, \ldots J_{j}$ and $K_{1}, \ldots K_{k}$ are disjoint.

3) Each $U_{I}$ is a (possibly empty) vector of bits, each of which is the mod-2 sum of a set (possibly singleton) of source components.

The full-strength version of Timo et al.'s result omits conditions 2) and 3) but replaces the rate expression in (21) with one that is more complex. Under conditions 2) and 3), however, their expression reduces to (21). Also Timo et al. state their result as an upper bound on $R(D)$ defined in Section II as opposed to $R_{o p t}$ as defined in Section IV. That is, they provide a guarantee on the expected time-average distortion, instead of on the block error probability that we use to define index coding. Their proof technique can be used to bound the block error probability with minimal modification, however.

One way of interpreting $U_{I}$ is that it is a "message" that is "sent" to all Decoders $i$ such that $i \in I$. That is, $U_{I}$ includes some information about the source that is decoded by all of the decoders in $I$ but is not available to any of the decoders in $I^{c}$. The contribution of $U_{I}$ to the overall rate in 21] is simply the rate needed to send $U_{I}$ to all of the decoders in $I$ using standard binning arguments (and relying on the fact that $U_{I}$ is a deterministic function of the source $\mathbf{S}$ ).

Evaluating this upper bound requires finding the optimal joint distribution of the $U_{I}$ auxiliary random variables. Since each $U_{I}$ is a deterministic function of $\mathbf{S}$, this is equivalent to finding the optimal such functions. Such an optimization problem is evidently quite complicated. We shall provide a polynomial-time heuristic for finding a feasible choice of the $U_{I} \mathrm{~s}$. Of the many different index coding schemes that have been proposed (e.g., [4], [2], [6], [7], [8] ), ours most closely resembles the partition multicast of Tehrani, Dimakis, and Neely [6]. In the language of our setup, their scheme amounts to finding the optimal choice of the $U_{I}$ subject to the constraint that each $U_{I}$ must be a vector consisting of a (possibly empty) subset of the source components. Tehrani et al. show that finding this optimal choice is NP-hard [6]. Our scheme, in contrast, consists of three steps, the first two of which amount to a polynomial-time heuristic for finding a reasonable and feasible (but not necessarily optimal) choice of auxiliary random variables subject to the constraint that each $U_{I}$ must be a vector consisting of a subset of the source components. Thus the output of the second step of our heuristic is a feasible solution to the optimization problem for which partition multicast is optimal. Our third step, however, replaces some of the $U_{I}$ variables with ones that are more general functions of source, i.e., not just subsets of the source variables. Due to the similarity between our heuristic and

\footnotetext{
${ }^{4}$ The weakened form of this result that we stated in the conference version of this work [27] was invalid in that it omitted one of the necessary conditions on the auxiliary random variables. The result has been corrected here.
} 
partition multicast, we call our heuristic coded approximate partition multicast (CAPM). Although CAPM is not guaranteed to be never worse than partition multicast, we shall show that it is optimal for all of the explicit scenarios for which Tehrani $e t$ al. show that partition multicast is optimal as well as some other, more general scenarios.

\section{CAPM: SElEction of $U_{I}$ 's IN THE ACHIEVABlE SCHEME FOR INDEX Coding}

CAPM is a method for choosing a feasible choice of the auxiliary random variables $U_{I}$ for $I \subseteq[m]$. Note that the number of auxiliary random variables is exponential in the number of decoders, although in typical instances most of these random variables will be null. To minimize the worst-case complexity of CAPM, therefore, we shall work with a linked list of the auxiliary random variables that are not null, which shall begin empty. We shall call all $U_{I}$ auxiliary random variables for which $|I|=i$ "level $i$ messages."

Step 1 : Beginning with an empty linked list of auxiliary random variables, we sequence through the vector of source bits. Any given bit must be in $f_{K J}$ for some $K \subseteq J \subseteq[m]$. So long as $J \neq[m]$, we seek to include this bit in $U_{K \cup J^{c}}$ : if $U_{K \cup J^{c}}$ does not exist in our linked list of auxiliary random variables, then we add it to the list and set it equal to the source bit in question. If it already exists in the list, then we locate it, and we set $U_{K \cup J^{c}}$ to be a vector of bits consisting of all source bits that were included previously along with this newly included source bit. For a source bit in $f_{K J}$ where $J=[m]$, we include the bit in the auxiliary random variable $U_{[m]}$, i.e., the auxiliary random variable that is decoded by all of the decoders. This process is repeated until all of the source bits have been included in an auxiliary random variable. Note that each nonvoid auxiliary random variable is then simply a vector of source bits. Also note that each source bit will be included in exactly one auxiliary random variable.

We now sort the linked list so that all level-2 messages appear first, followed by all level-3 messages, etc. Note that all level-1 messages are necessarily empty (assuming there is more than one decoder), by virtue of the fact that every source bit is assumed to be demanded by at least one decoder, and source bits that no decoder has as side information are placed in $U_{[m]}$. The complexity of Step 1 is at most $O\left(s^{2} \cdot m\right)$, where $s=|\mathbf{S}|$.

Remark 4. See Proposition 3 to follow for a justification of this particular approach to allocating the source components among the different auxiliary random variables.

Step 2 : Let $U_{I}$ denote the first auxiliary random variable in the linked list. If $I=[m]$, i.e., this first auxiliary random variable is decoded by all of the decoders, then this $U_{I}$ must be the only nonnull auxiliary random variable (since they are sorted by level), in which case we skip Step 2 and proceed to Step 3. Suppose instead that $|I|<m$. Note that $U_{I}$ 's contribution to the overall rate is

$$
\max _{i \in I} H\left(U_{I} \mid \mathbf{Y}_{\mathbf{i}}\right)
$$

In many cases $H\left(U_{I} \mid \mathbf{Y}_{\mathbf{i}}\right)$ will not be constant over $i \in I$. That is, some decoders in $I$ will require a higher rate to decode $U_{I}$ than others. When this happens we move some of the source bits in $U_{I}$ to a higher-level message. Define the two decoder indices

$$
i^{*}=\min \left\{i: H\left(U_{I} \mid \mathbf{Y}_{\mathbf{i}}\right)=\min _{l \in I} H\left(U_{I} \mid \mathbf{Y}_{\mathbf{l}}\right)\right\}
$$

and

$$
j^{*}=\min \left\{j: H\left(U_{I} \mid \mathbf{Y}_{\mathbf{j}}\right)=\max _{l \in I} H\left(U_{I} \mid \mathbf{Y}_{\mathbf{l}}\right)\right\}
$$

If $H\left(U_{I} \mid \mathbf{Y}_{\mathbf{i}^{*}}\right)<H\left(U_{I} \mid \mathbf{Y}_{\mathbf{j}^{*}}\right)$, then there must exist a source bit in $U_{I}$ that is contained in $\mathbf{Y}_{\mathbf{i}^{*}}$ but not in $\mathbf{Y}_{\mathbf{j}^{*}}$. We select the lowest-index source bit with this property and move it from $U_{I}$ to some $U_{J}$ such that $I \subset J$ and $|J|=|I|+1$. If $|I|<m-1$, then there are many such choices of $J ; J$ can be chosen arbitrarily, but for concreteness we shall assume the following. First we look for nonempty $U_{J}$ 's such that $I \subset J$ and $|J|=|I|+1$. If we can find such a message or messages, we select the $J$ with the lowest index that is not already in $I$. If that is not the case, $J$ is obtained by adding to $I$ the lowest index that is not already in $I$. We call the bit that is moved leftover or excess. We then recompute $i^{*}$ and $j^{*}$ according to (22) and (23), respectively, and move an additional bit to a higher-level message if necessary, repeating this process until $U_{I}$ is such that $H\left(U_{I} \mid \mathbf{Y}_{\mathbf{i}^{*}}\right)=H\left(U_{I} \mid \mathbf{Y}_{\mathbf{j}^{*}}\right)$. Note that this condition must eventually be satisfied, since after sufficiently many iterations, $U_{I}$ will become null. Once this condition is satisfied for $U_{I}$, we apply the same procedure to the next auxiliary random variable in the linked list, and so on until this procedure has been applied to every variable in the linked list. It is possible that some auxiliary random variables in the linked list are made null through this procedure, in which case they are removed from the linked list. The complexity of Step 2 is $O\left(m^{2} \cdot s^{3}\right)$.

Remark 5. The rationale for moving source bits up to higher-level messages is as follows. A bit that is excess contributes to the maximum

$$
\max _{l \in I} H\left(U_{I} \mid \mathbf{Y}_{\mathbf{l}}\right),
$$


which is $U_{I}$ 's contribution to the overall rate. Thus removing this bit from $U_{I}$ has the potential to reduce $U_{I}$ 's contribution to the rate (although it will not necessarily do so, if there are multiple l that achieve the maximum in (24); see the next remark). Of course, including this bit in a higher-level message, $U_{J}$, will tend to increase $U_{J}$ 's contribution to the rate. But it will only do so the source bit in question is not in the side information of one of the decoders $l$ that achieve the maximum in

$$
\max _{l \in J} H\left(U_{I} \mid \mathbf{Y}_{\mathbf{J}}\right) \text {. }
$$

Thus moving the bit up one level often yields a rate reduction, and even if it does not, it may yield a rate reduction upon being elevated again during a later iteration.

Remark 6. If there exists a unique $j \in I$ such that $H\left(U_{I} \mid \mathbf{Y}_{\mathbf{j}}\right)=\max _{l} H\left(U_{I} \mid \mathbf{Y}_{\mathbf{l}}\right)$, then moving an excess bit to a higher-level message cannot increase the overall rate, and in some cases it may strictly decrease the rate. If the decoder with maximum rate is not unique, then moving an excess bit to a higher-level message can increase the rate, as in Example 1 to follow, although this increase is sometimes offset during later movements of excess bits, or during Step 3 (again as in Example 17). For this reason we move excess bits according to the procedure outlined in Step 2 even when such movements have the immediate effect of increasing the overall rate.

Remark 7. Finding the feasible allocation of source components among the various $U_{I}$ variables that minimizes the rate in (21]) is NP-hard, as shown by Tehrani et al. [6].

Step 3 : In the final step, we exclusive-OR (XOR) some of the bits included in the auxiliary random variables. Let $U_{I}$ denote the first auxiliary random variable in the linked list, and suppose that $V_{1}, \ldots, V_{l}$ denote the excess source bits that are included in $U_{I}$. Recall that bits placed in $U_{[m]}$ during Step 1 are not considered excess. For each $i$, let $N_{i}$ denote the set of decoders that need (i.e., demand) $V_{i}$ and let $H_{i}$ denote the set of decoders that have $V_{i}$ has side information. We search for a pair of components $V_{i}$ and $V_{j}$ such that $N_{i} \subset H_{j}, N_{j} \subset H_{i}$, and $V_{i}$ and $V_{j}$ were included in the same auxiliary random variable in Step 1 (that is, $N_{i} \cup H_{i}=N_{j} \cup H_{j}$ ). If there are no such $V_{i}$ and $V_{j}$ then we proceed to the next $U$ variable in the linked list. Otherwise, we delete $V_{j}$ from $U_{I}$, we replace $V_{i}$ in $U_{I}$ with $V_{i} \oplus V_{j}$, we replace $N_{i}$ with $N_{i} \cup N_{j}$ and $H_{i}$ with $H_{i} \cap H_{j}$. Since both $V_{i}$ and $V_{j}$ were placed in the same auxiliary random variable in Step 1, we view the new $V_{i}$ as also being placed in that variable in Step 1, although of course the auxiliary random variables constructed in Step 1 did not involve taking the XOR of any of the source components. We then repeat this process, again looking for $V_{i}$ and $V_{j}$ such that $N_{i} \subset H_{j}, N_{j} \subset H_{i}$, and $V_{i}$ and $V_{j}$ were included in the same auxiliary random variable in Step 1. If we find such a pair, we replace them with their exclusive-OR. We repeat this process until there are no such pairs remaining. We then apply this procedure to all of the other auxiliary random variables in the linked list. The complexity of Step 3 is $O\left(m \cdot s^{3}\right)$.

Remark 8. Evidently Step 3 will never increase the rate.

Remark 9. One could certainly exclusive-OR bits $V_{i}$ and $V_{j}$ satisfying $N_{i} \subset H_{j}$ and $N_{j} \subset H_{i}$ but for which $V_{i}$ and $V_{j}$ are not included in the same auxiliary random variable in Step 1 or for which either $V_{i}$ or $V_{j}$ are not excess bits. Choosing to exclusive-OR certain pairs of bits can foreclose other such choices, however, and the latter choices may ultimately lead to lower rates. The restriction that we only exclusive-OR bits that are excess and that originated in the same auxiliary random variable in Step 1 is intended to guide the process toward the most productive exclusive-OR choices. Of course, once the above process exhausts all of its exclusive-OR possibilities, one could look for exclusive-OR opportunities among bits that are not excess or that did not originate in the same auxiliary random variable. We shall not include this step in the heuristic, however, since it is not necessary in any of our optimality results or any of our examples.

One can verify that this selection procedure provides a feasible choice of the $U_{I}$ variables as follows. First note that the choice will be feasible after each step 1 . This is because each source component is included in a $U_{I}$ variable that is decoded by all of the decoders that demand it. Thus condition 1) in Proposition 1 is satisfied. Conditions 2) and 3) are satisfied because each $U_{I}$ consists of a subset of the source components and these subsets are disjoint across the $U_{I}$ s. Step 2 only moves source components from a $U_{I}$ to a $U_{J}$ for which $I \subseteq J$, so it is evident that conditions 1)-3) continue to hold. Finally, the exclusive-OR operation applied in Step 3 evidently never violates conditions 2) or 3), and the specific conditions under which the exclusive-OR operation is applied ensures that condition 1) continues to hold.

Definition 8. The achievable rate provided by CAPM is denoted by $R_{\mathrm{CAPM}}$.

To illustrate CAPM, we provide two examples.

Example 1. Consider the 4-decoder index coding problem instance with demands $f_{12^{c}}, f_{32^{c}}, f_{3\{1,2\}^{c}}, f_{1\{2,3\}^{c}}, f_{4[4]}, f_{2[4]}$ where each demand is one bit and $a^{\mathrm{c}}=[\mathrm{m}] \backslash\{a\}$. Now we show each step of CAPM.

Step 1: At the end of this step we have

$U_{12}=f_{12^{c}}, U_{23}=f_{32^{c}}$,

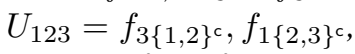

$U_{1234}=f_{4[4]}, f_{2[4]}$. 
Step 2: We start with level-2 messages. The first level-2 message is $U_{12} . f_{12}$ in $U_{12}$ is an excess bit and since we already have level-3 message $U_{123}$ which $f_{12^{c}}$ can be placed we move $f_{12^{c}}$ to $U_{123}$. The next message is $U_{23} . f_{32^{c}}$ in $U_{23}$ is an excess bit and it is also placed to $U_{123}$. The messages at this point are

$U_{123}=f_{3\{1,2\}^{c}}, f_{1\{2,3\}^{c}}, f_{12^{c}}, f_{32^{c}}$,

$U_{1234}=f_{4[4]}, f_{2[4]}$

and we move on to level-3 messages. Note that there is only one level-3 message, $U_{123}$. All demands in it are excess bits since $H\left(U_{123} \mid \mathbf{Y}_{2}\right)=0$. We move all excess bits to $U_{1234}$, which is the only one level-4 message. This completes the Step 2 and we have

$U_{1234}=f_{4[4]}, f_{2[4]}, f_{3\{1,2\}^{c}}, f_{1\{2,3\}^{c}}, f_{12^{c}}, f_{32^{c}}$

at the end of this step.

Step 3: Note that $\left(f_{3\{1,2\}^{\mathrm{c}}}, f_{1\{2,3\}^{\mathrm{c}}}\right)$ are the only excess bits that were in the same message at Step 1 and $f_{3\{1,2\}^{\mathrm{c}}} \oplus f_{1\{2,3\}^{\mathrm{c}}}$ is decodable at the respective decoders. Hence at the end of Step 3, selection of the messages is the following:

$U_{1234}=f_{4[4]}, f_{2[4]}, f_{3\{1,2\}^{\mathrm{c}}} \oplus f_{1\{2,3\}^{\mathrm{c}}}, f_{12^{\mathrm{c}}}, f_{32^{\mathrm{c}}}$

and all others are empty.

Note that after Step 2 the total rate is 6 bits, whereas after Step 3 the rate is 5 bits. The lower bound in Theorem 2 also gives 5 bits, showing that CAPM achieves the optimal rate for this example.

Remark 10. Let $R_{a}$ be a rate obtained by placing $f_{I J}$ 's in messages ( $U_{K}$ 's) by applying Step 1 . Let $R_{a}^{*}$ be a rate obtained such that $f_{I J}$ 's are placed in messages ( $U_{K}^{*}$ 's) by following the Step 1 , and applying the Step 2 only for level- 2 messages.

Note that $U_{K}=U_{K}^{*}$, for all level-i messages where $i>3$. Also, each possible excess bit (or bits) which we will denote by $f_{I J}^{*} \subseteq f_{I J}$, coming from a level-2 message $U_{K}^{*}$ is such that either $I=\{\alpha\}$ or $I=\{\beta\}$ where $K=\{\alpha, \beta\}$. Then, when level-2 messages $U_{K}$ and $U_{K}^{*}$, where $K=\{\alpha, \beta\}$, are not the same, we can write $U_{K}^{*} \cup f_{I J}^{*}=U_{K}$ and

$$
\begin{aligned}
& \max _{i \in K}\left\{H\left(U_{K} \mid \mathbf{Y}_{\mathbf{i}}\right)\right\}=\max _{i \in K}\left\{H\left(U_{K}^{*} \cup f_{I J}^{*} \mid \mathbf{Y}_{\mathbf{i}}\right)\right\} \\
& =\max _{i \in K}\left\{H\left(U_{K}^{*} \mid \mathbf{Y}_{\mathbf{i}}\right)+H\left(f_{I J}^{*} \mid \mathbf{Y}_{\mathbf{i}}\right)\right\} \\
& \stackrel{a}{=} H\left(U_{K}^{*} \mid \mathbf{Y}_{\mathbf{i}}\right)+\max _{i \in K}\left\{H\left(f_{I J}^{*} \mid \mathbf{Y}_{\mathbf{i}}\right)\right\} \\
& \stackrel{b}{=} H\left(U_{K}^{*} \mid \mathbf{Y}_{\mathbf{i}}\right)+H\left(f_{I J}^{*}\right)
\end{aligned}
$$

a: Since all $H\left(U_{K}^{*} \mid \mathbf{Y}_{\mathbf{i}}\right)$ for $i \in K$ are the same.

b: Since $f_{I J}^{*}$ is such that either $I=\{\alpha\}$ or $I=\{\beta\}$.

Hence, we can write $R_{a}-R_{a}^{*}$ as

$$
\begin{aligned}
& \left(\sum_{|I|=3} \max _{i \in I}\left\{H\left(U_{I} \mid \mathbf{Y}_{\mathbf{i}}\right)\right\}-\sum_{|I|=3} \max _{i \in I}\left\{H\left(U_{I}^{*} \mid \mathbf{Y}_{\mathbf{i}}\right)\right\}\right)+\left(\sum_{|K|=2} \max _{i \in K}\left\{H\left(U_{K} \mid \mathbf{Y}_{\mathbf{i}}\right)\right\}-\sum_{|K|=2} \max _{i \in K}\left\{H\left(U_{K}^{*} \mid \mathbf{Y}_{\mathbf{i}}\right)\right\}\right) \\
= & \left(\sum_{|I|=3} \max _{i \in I}\left\{H\left(U_{I} \mid \mathbf{Y}_{\mathbf{i}}\right)\right\}-\sum_{|I|=3} \max _{i \in I}\left\{H\left(U_{I}^{*} \mid \mathbf{Y}_{\mathbf{i}}\right)\right\}\right)+\sum_{f_{I J}^{*}} H\left(f_{I J}^{*}\right), \quad \text { from 25). }
\end{aligned}
$$

Since $U_{I}^{*}=U_{I} \cup f_{I}^{*}$ where $f_{I}^{*}$ denotes all of the excess bits in $U_{I}^{*}$ and

$$
\max _{i \in I}\left\{H\left(U_{I} \cup f_{I}^{*} \mid \mathbf{Y}_{\mathbf{i}}\right)\right\} \leq \max _{i \in I}\left\{H\left(U_{I} \mid \mathbf{Y}_{\mathbf{i}}\right)\right\}+H\left(f_{I}^{*}\right),
$$

we can write

$$
R_{a}-R_{a}^{*} \geq-\sum_{|I|=3} H\left(f_{I}^{*}\right)+\sum_{f_{I J}^{*}} H\left(f_{I J}^{*}\right)=0 .
$$

Note that since we apply Step 2 once to the level-2 messages, the leftover bits that we get are unique, i.e., independent of the sorted demand sequence given at the beginning of Step 1 and different leftover bits coming from previous levels (since there is no leftover bit coming to level-2 messages). However, if we apply CAPM for an arbitrary instance of an index coding problem, this may not be the case. In other words, at Step 2 of the CAPM, we may get different excess bits due to differently sorted demand sequence given at the beginning of Step 1 or different leftover bits coming from previous levels and this may affect the resulting rate. Also, when there are multiple options for leftover bits to be moved, one may get different rates due to the selection of different next level messages to move the leftover bits. Lastly, there may be instances of index coding problem where not moving the bits to the next level gives a lower rate. To illustrate some of these issues, we provide the following examples. For the examples, $f_{I J}^{a} \backslash U_{K}$ denotes the leftover bits ( $a$ bits) of $f_{I J}$ from $U_{K}$. If all $f_{I J}$ are leftover bits then we remove the superscript $a$. 
Example 2. Consider the 4-decoder index coding problem instance with demands $f_{12^{c}}, f_{23^{c}}, f_{31^{c}}, f_{3\{1,2\}^{c}}, f_{4[4]}$ where $f_{12^{c}}, f_{23^{c}}$ are two bits and the rest are one bit. Now, we explain each step of the CAPM for this example.

Step 1: At the end of this step we have

$U_{12}=f_{12^{c}}, U_{23}=f_{23^{c}}, U_{13}=f_{31^{c}}$,

$U_{123}=f_{3\{1,2\}}$, $U_{1234}=f_{4[4]}$.

Step 2: We begin with level-2 messages. Note that all demands at level-2 messages are excess bits and can be moved to level-3 message, $U_{123}$. Then we have,

$U_{123}=f_{3\{1,2\}^{c}}, f_{12^{c}}, f_{23^{c}}, f_{31^{c}}$,

$U_{1234}=f_{4[4]}$.

We move on to level-3 messages. There is only one level-3 message, $U_{123}$, and one bit of $f_{12^{c}}$, denoted as $f_{12^{c}}^{1}$, is an excess bit. Then we move it to $U_{1234}$, concluding Step 2. Hence, messages at the end of this step are

$U_{123}=f_{3\{1,2\}^{c}}, f_{12^{c}}^{1}, f_{23^{c}}, f_{31^{c}}$,

$U_{1234}=f_{4[4]}, f_{12^{\mathrm{c}}}^{1}$.

Step 3: Since there is no $\oplus$ opportunity as described in Step 3, the messages at the end of Step 2 remains the same, giving a total rate of 5 bits.

Note that without loss of generality we can label Decoder 3 as 1 and Decoder 1 as 3. Then, if we apply CAPM with this relabeling we get the following messages at each step.

Step 1: At the end of this step we have

$U_{32}=f_{32^{c}}, U_{12}=f_{21^{c}}, U_{13}=f_{13^{c}}$,

$U_{123}=f_{1\{2,3\}^{\mathrm{c}}}, U_{1234}=f_{4[4]}$.

Step 2: We begin with level-2 messages. Similar to previous case all demands at level-2 messages are excess bits and moved to $U_{123}$. Then we have,

$U_{123}=f_{1\{2,3\}^{c}}, f_{32^{c}}, f_{21^{c}}, f_{13^{c}}$,

$U_{1234}=f_{4[4]}$.

We move on to level-3 messages. As in the previous case, there is only one level-3 message, $U_{123}$. However, now the excess bits of $U_{123}$ are $f_{1\{2,3\}^{c}}, f_{21^{c}}^{1}, f_{32^{c}}^{1}$. Then we move these to $U_{1234}$, concluding Step 2 . Hence, messages at the end of this step are

$U_{123}=f_{32^{c}}^{1}, f_{21^{c}}^{1}, f_{13^{c}}$,

$U_{1234}=f_{4[4]}, f_{1\{2,3\}^{c}}, f_{21^{c}}^{1}, f_{32^{c}}^{1}$,

Step 3: Since there is no $\oplus$ opportunity as described in Step 3, the messages at the end of Step 2 remains the same, giving a total rate of 6 bits. Thus rate achieved by the heuristic depends on the indexing of the decoders.

Example 3. Consider the 5-decoder index coding problem instance with demands $f_{1[5]}, f_{5[5]}, f_{2\{1,4\}^{\mathrm{c}}}, f_{3\{1,2\}^{\mathrm{c}}}, f_{4\{1,3\}^{\mathrm{c}}}$ where each demand is one bit. Now we show each step of the CAPM.

Step 1: At the end of this step we have,

$U_{124}=f_{2\{1,4\}^{\mathrm{c}}}, U_{123}=f_{3\{1,2\}^{\mathrm{c}}}, U_{134}=f_{4\{1,3\}^{\mathrm{c}}}$,

$U_{12345}=f_{1[5]}, f_{5[5]}$.

Step 2: We begin with the lowest level, i.e., level-3 for this example. Note that all of the demands in level-3 messages are excess bits and they are moved to $U_{1234}$. Then we have

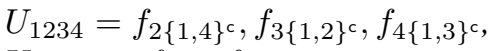

$U_{12345}=f_{1[5]}, f_{5[5]}$.

Note that total rate is 4 bits at this state. We move on to level-4 messages. There is only one level-4 message, $U_{1234}$ Since $H\left(U_{1234} \mid \mathbf{Y}_{\mathbf{1}}\right)=0$, all demands in $U_{1234}$ are excess bits and they are moved to $U_{12345}$. Then we have

$U_{12345}=f_{1[5]}, f_{5[5]}, f_{2\{1,4\}^{c}}, f_{3\{1,2\}}, f_{4\{1,3\}}$, where the total rate is 5 bits.

Step 3: Since there is no $\oplus$ opportunity as described in Step 3, the messages at the end of Step 2 remains the same and total rate is 5 bits. Note that if we did not move the excess bits at level-4 message, the total rate would be 4 bits.

In the next section, we show that applying CAPM gives us the optimal rate for several specific cases of the index coding problem.

\section{OPTIMALITy RESUlTS FOR INDEX CODING}

We shall show that CAPM yields the optimal rate for several scenarios. Since the partition multicast scheme of Tehrani et al. [6] is the most direct antecedent of CAPM, we begin by showing that CAPM coincides with the DSM+ lower bound, and is thus optimal, for all of the explicit scenarios for which Tehrani et al. show that partition multicast is optimal.

First, consider the case depicted in Fig. 2, in which there are $m$ decoders, $m$ source bits, and Decoder $k$ demands source bit $k$ and has source bit $k+1$ as side information, for $k \in\{1, \ldots, m-1\}$. Decoder $m$ demands source bit $m$ and has the first source bit as side information. Such an instance is typically called a "directed cycle" after its graph-theoretic description. 


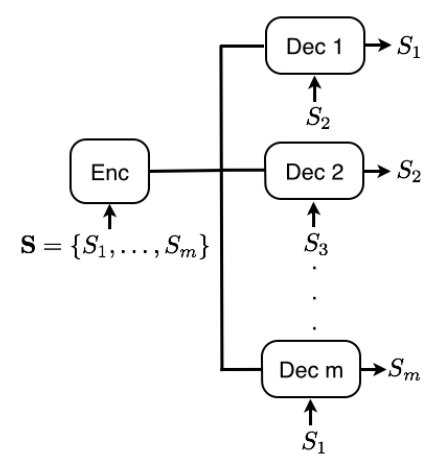

Fig. 2. Index coding instance which is also called "directed cycle"

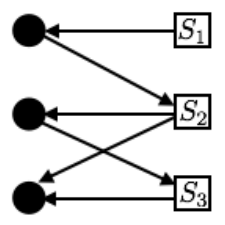

Fig. 3. Bipartite graph representation of index coding example with $m=3$ and $\mathbf{S}=\left\{S_{1}, S_{2}, S_{3}\right\}$. Circle nodes represent users while square nodes denote source bits.

Proposition 2. For the instance depicted in Fig. 2, the achievable rate provided by CAPM and the lower bound provided by the DSM+ bound coincide. In fact

$$
R_{\mathrm{CAPM}}=R_{D S M+}=m-1 .
$$

Proof: First we show that $R_{\mathrm{CAPM}}=m-1$. After Step 1 of CAPM, the messages are $U_{\{i, i+1\}}=S_{i+1}$ for all $i \in[m-1]$ and $U_{\{1, m\}}=S_{1}$. Observe that at any point of the algorithm, for any non-empty message $U_{I}$ where $\left|U_{I}\right|=k$, there exists $\mathbf{Y}_{\mathbf{j}} \in I$ such that $H\left(U_{I} \mid \mathbf{Y}_{\mathbf{j}}\right)=k-1$ and $k \geq H\left(U_{I} \mid \mathbf{Y}_{\mathbf{i}}\right) \geq k-1$ for all $i \in I$. Hence after Step 2 of CAPM, $H\left(U_{I} \mid \mathbf{Y}_{\mathbf{j}}\right)$ will be equal to $k-1$ for all $j \in I$ for any nonempty message $U_{I}$. Now we show that for any non-empty message $U_{I}$ with $\left|U_{I}\right|=k, H\left(U_{I} \mid \mathbf{Y}_{\mathbf{j}}\right)=k-1$ for all $j \in I$ if and only if $\left|U_{I}\right|=m$. This will imply that $R_{\mathrm{CAPM}} \leq m-1$. Consider a $U_{I}$ such that $\left|U_{I}\right|=k$ and $H\left(U_{I} \mid \mathbf{Y}_{\mathbf{j}}\right)=k-1$ for all $j \in I$. Let $i=\min \left\{j: S_{j} \in U_{I}\right\}$. Then by virtue of Steps 1) and 2) of CAPM, we must have $i \in I$. Since $H\left(U_{I} \mid \mathbf{Y}_{\mathbf{i}}\right)=k-1$, we must have $S_{(i+1)} \in U_{I}$ as well, where $(j)=((j-1) \bmod m)+1$. Likewise, $(i+1) \in I$, which implies that $(i+2) \in I$, etc. It follows, then, that $|I|=\left|U_{I}\right|=m$.

Conversely, selecting the permutation $\sigma(i)=m-i+1$ in Theorem 2 shows that $R_{D S M+} \leq m-1$.

We can represent any (groupcast) index coding problem as a bipartite graph $G=(\mathbf{M}, \mathbf{S}, E)$ where $\mathbf{M}, \mathbf{S}$, and $E$ denote the set of user nodes, the set of source bit nodes and the set of edges respectively [6]. There is a directed edge $\left(m_{i}, S_{i}\right), m_{i} \in \mathbf{M}$, $S_{i} \in \mathbf{S}$ if and only if $m_{i}$ has $S_{i}$ as side information (i.e., $\left.S_{i} \in \mathbf{Y}_{\mathbf{m}_{\mathbf{i}}}\right)$ and there is a directed edge $\left(S_{i}, m_{i}\right), S_{i} \in \mathbf{S}, m_{i} \in \mathbf{M}$ if and only if $m_{i}$ demands $S_{i}$ (i.e., $S_{i} \in \mathbf{f}_{\mathbf{m}_{\mathbf{i}}}$ and see Figure 3 for an example).

Second, we consider an index coding instance represented by a directed acyclic graph (DAG). Tehrani et al. [6] show that partition multicast achieves the optimal rate for DAGs and the optimal rate equals to total number of demanded bits, $s=|\mathbf{S}|$. Note that for any given instance of an index coding problem, the rate achieved by CAPM cannot be more than the number of demanded bits. Thus it suffices to show that the rate $s$ is optimal. Tehrani et al. show this under the zero-error formulation. Using the DSM+ lower bound, one can show that the optimal rate is also $s$ under the vanishing block error probability assumption.

Lemma 3. For an instance of the index coding problem represented by a DAG, there exists a permutation, $\sigma(\cdot)$, on $[m]$ such that

$$
\mathbf{Y}_{\sigma(\mathbf{i})} \subseteq \cup_{j=1}^{i-1} \mathbf{f}_{\sigma(\mathbf{j})}, \text { for all } i \in[m] .
$$

Proof: We follow Neely et al. [28]. Observe that every index coding problem represented by a DAG must have a node in the graph with no outgoing edges. This node must represent some decoder $\ell$ since every source bit is assumed to be demanded by at least one decoder (see Section IV). Decoder $\ell$ must then have no side information. Let $\sigma(1)=\ell$.

We then proceed by induction. Suppose the containment in $[26$ holds for all $i$ in $[k]$ with $k<m$. Consider the modified index coding instance in which we delete Decoders $\sigma(1), \ldots, \sigma(k)$ and all of their incoming and outgoing edges in the graph. We also delete any source components that are left with no edges. This instance must again be a DAG, and since $k<m$ it 
must have at least one decoder node, so there must be a decoder $\nu$ that has no side information. It follows that in the original instance, $\mathbf{Y}_{\nu} \subset \cup_{j=1}^{k} \mathbf{f}_{\sigma(j)}$. We then set $\sigma(k+1)=\nu$.

By Lemma 3, we have that the DSM+ lower bound $R_{D S M+}$ is greater than or equal to

$$
\begin{aligned}
& H\left(\mathbf{f}_{\sigma(\mathbf{1})} \mid \mathbf{Y}_{\sigma(\mathbf{1})}\right)+H\left(\mathbf{f}_{\sigma(\mathbf{2})} \mid \mathbf{f}_{\sigma(\mathbf{1})}, \mathbf{Y}_{\sigma(\mathbf{1})}, \mathbf{Y}_{\sigma(\mathbf{2})}\right)+\cdots+H\left(\mathbf{f}_{\sigma(\mathbf{m})} \mid \mathbf{f}_{\sigma(\mathbf{1})}, \ldots, \mathbf{f}_{\sigma(\mathbf{m}-\mathbf{1})}, \mathbf{Y}_{\sigma(\mathbf{1})}, \ldots, \mathbf{Y}_{\sigma(\mathbf{m})}\right) \\
& =H\left(\mathbf{f}_{\sigma(\mathbf{1})}\right)+H\left(\mathbf{f}_{\sigma(\mathbf{2})} \mid \mathbf{f}_{\sigma(\mathbf{1})}\right)+\cdots+H\left(\mathbf{f}_{\sigma(\mathbf{m})} \mid \mathbf{f}_{\sigma(\mathbf{1})}, \ldots, \mathbf{f}_{\sigma(\mathbf{m}-\mathbf{1})}\right),
\end{aligned}
$$

giving $R_{D S M+} \geq s$. Hence CAPM gives the optimal rate for DAGs.

Finally, Tehrani et al. [6] show that partition multicast achieves the optimal rate when each decoder demands a single bit and has as side information all of the other source bits. Note that under these assumptions one may, without loss of generality, assume that each source bit is demanded by at most one decoder; two decoders that demand the same source bit must have the same side information and therefore one of the two can be deleted without affecting the rate. Then each source component must be present as side information at either all or all but one of the decoders.

We shall prove that CAPM is optimal for the more general scenario in which each source bit is present at none of the decoders, all of the decoders, all but one, or all but two. That is, $\mathbf{S}$ consists of $\left\{G_{0}, G_{m}, G_{m-1}, G_{m-2}\right\}$. We do not assume that each decoder demands a single bit or that each bit is demanded by at most one decoder.

Theorem 3. The optimal rate, $R_{\text {opt }}$, for the index coding problem where $\mathbf{S}=\left\{G_{0}, G_{m}, G_{m-1}, G_{m-2}\right\}$ is

$$
R_{o p t}=\max \left\{R_{1}, \ldots, R_{m}\right\}
$$

where

$$
\begin{aligned}
R_{i}= & H\left(\mathbf{f}_{\mathbf{1}} \backslash\left\{\bigcup_{\{1, \beta\} \subseteq[m]} f_{1\{1, \beta\}}\right\}, \ldots, \mathbf{f}_{\mathbf{i}-\mathbf{1}} \backslash\left\{\cup_{\{i-1, \beta\} \subseteq[m]} f_{i-1\{i-1, \beta\}}\right\},\right. \\
& \left.\mathbf{f}_{\mathbf{i}}, \mathbf{f}_{\mathbf{i}+\mathbf{1}} \backslash\left\{\bigcup_{\{i+1, \beta\} \subseteq[m]} f_{i+1\{i+1, \beta\}}\right\}, \ldots, \mathbf{f}_{\mathbf{m}} \backslash\left\{\bigcup_{\{m, \beta\} \subseteq[m]} f_{m\{m, \beta\}}\right\} \mid \mathbf{Y}_{\mathbf{i}}\right)+\max _{j, j \in[m] \backslash i} f_{j\{i, j\}},
\end{aligned}
$$

and is achieved by CAPM.

Corollary 1. For any index coding problem with three or fewer decoders, the optimal rate is given by (27), and is achieved by CAPM.

Proof: Any index coding problem with three or fewer decoders must have the property that each source component is present as side information at either all of the decoders, none of the decoders, all but one, or all but two.

Remark 11. Since CAPM and the DSM+ lower bound only give integer-valued bounds, it follows that the optimal rate is integer-valued for the scenario described in Theorem 3 , and in particular, in Corollary 1 .

Remark 12. Corollary 1 solves the index coding problem with three decoders and any number of source components. In contrast, Arbabjolfaei et al. [8] solve the index coding problem with up to five source components and any number of decoders. Evidently neither of these results implies the other, even if one ignores slight differences in the problem formulation between the two papers. It is also worth noting that the Arbabjolfaei et al. result is numerical while Corollary 1 is analytical.

Proof of Theorem 3 .

1. Achievability:

We utilize the achievable scheme of Proposition 1 and apply the CAPM for selection of $U_{I}$ to get an explicit expression. Step 1: Note that there is no demand related to $G_{m}$ since all decoders have it as side information. We place all demands of all decoders related to $G_{m-1}$ and $G_{0}$ into $U_{[m]}$. The remaining demands are subsets of $G_{m-2}$, i.e., components that $m-2$ of decoders have. For any $\mathbf{S}_{\mathbf{J}} \in G_{m-2}$, where $J=\{\alpha, \beta\}, \alpha \neq \beta$ Decoder $\alpha$ and Decoder $\beta$ are the two decoders that do not have $\mathbf{S}_{\mathbf{J}}$ as side information. Then we place $f_{\{\alpha, \beta\} J}$ to $U_{[m]}$. Also, we place $f_{\alpha J}$ and $f_{\beta J}$ to level $m-1$ messages. Since there is no demand related to $G_{i}, \forall i \in[m-3]$, all messages $U_{I},|I| \leq m-2$ will be empty. This completes the Step 1 .

Step 2 and 3: To determine the leftover bits in Step 2 and bits to be XORed in Step 3 we write the demands in the following way: Note that there are $m(m-1)$ different non overlapping pairs of demands related to $G_{m-2}$ since $\left|G_{m-2}\right|=\frac{m(m-1)}{2}$ and there are two demands $f_{\alpha J}$ and $f_{\beta J}$ for each $\mathbf{S}_{\{\alpha, \beta\}} \in G_{m-2}$. Also, note that for each Decoder $\alpha$ there are $m-1$ non overlapping demands, $f_{\alpha J}$ related to $G_{m-2}$. Therefore, we can put all those non overlapping demands into a matrix $A$ with $m$ rows and $m-1$ columns in the following way. $\alpha^{\text {th }}$ row, denoted by $A_{\alpha}$, consists of demands $f_{\beta\{\alpha, \beta\}}$ where $\beta$ runs over the set $[m] \backslash\{\alpha\}$. Note that $A_{\alpha}$ does not contain any demand from the Decoder $\alpha$. Also, for each $f_{\beta\{\alpha, \beta\}}$, all entries of $A_{\alpha}$ other than $f_{\beta\{\alpha, \beta\}}$ exist as side information at Decoder $\beta$. Hence, we observe that all non overlapping demands which are related to $G_{m-2}$ and placed in $U_{\alpha^{c}}$ at Step 1 are in $A_{\alpha}$. Also, there is no other type of demand in level $m-1$ messages.

Lastly, as the size of each demand in $A_{\alpha}$ can be different, we arrange the entries in $A_{\alpha}$ in an increasing order with respect to their sizes. If two demands are in equal size, which one is put first does not matter. This completes the construction of the matrix $A$.

For each $A_{\alpha}$ in $A$ we apply the following $\oplus$ operation. 
Definition 9. Let $a_{i}, i \in\{1, \ldots, m-1\}$ be vectors. Assume without loss of generality that $l_{1} \leq l_{2} \leq, \ldots, \leq l_{m-1}$ where $l_{i}=\left|a_{i}\right|$ denotes the number of elements in $a_{i}$. Then,

$$
\left(a_{1}, a_{2}, \ldots, a_{m-1}\right)^{\oplus}=\left(a_{1}^{\oplus}, \ldots, a_{m-1}^{\oplus}\right)
$$

where

$$
\begin{aligned}
\left(a_{1}^{\oplus}, \ldots, a_{m-1}^{\oplus}\right)= & \left(a_{1} \oplus\left(a_{2}\right)_{l_{1}} \oplus \cdots \oplus\left(a_{m-1}\right)_{l_{1}},\right. \\
& \left(a_{2}\right)_{l_{2}-l_{1}} \oplus \cdots \oplus\left(a_{m-1}\right)_{l_{2}-l_{1}}, \\
& \cdots, \\
& \left.\left(a_{m-1}\right)_{l_{m-1}-l_{m-2}}\right),
\end{aligned}
$$

and where $\left(a_{i}\right)_{l_{j}-l_{k}}$ denotes the vector consisting of components of $a_{i}$ from $\left(l_{k}+1\right)^{\text {th }}$ to $l_{j}^{\text {th }}$ component.

Note that all the components in $A_{\alpha 2}^{\oplus}, \ldots, A_{\alpha(m-1)}^{\oplus}$ are leftover bits and moved to $U_{[m]}$. Then for each $A_{\alpha}^{\oplus}=$ $\left(A_{\alpha 1}^{\oplus}, \ldots, A_{\alpha(m-1)}^{\oplus}\right)$, all the components in $A_{\alpha 1}^{\oplus}$ (i.e., $\left.A_{\alpha 1},\left(A_{\alpha 2}\right)_{\left|A_{\alpha 1}\right|}, \ldots,\left(A_{\alpha(m-1)}\right)_{\left|A_{\alpha 1}\right|}\right)$ remain in $U_{\alpha^{c}}$. This concludes Step 2.

Lastly by Step 3, we have $U_{\alpha^{c}}=A_{\alpha 1}^{\oplus}$, for all $\alpha \in[m]$, and $A_{\alpha 2}^{\oplus}, \ldots, A_{\alpha(m-1)}^{\oplus}$ are in $U_{[m]}$. This concludes CAPM. Also for the ease of notation, when we write $A^{\oplus} \backslash\left\{\cup_{\alpha \in[m]} A_{\alpha 1}^{\oplus}\right\}$, we will mean the vector $A^{\oplus}$ with the components $A_{\alpha 1}^{\oplus}$ for all $\alpha \in[m]$ removed.

Hence,

$$
\begin{aligned}
& U_{[m]}=\mathbf{f}_{\mathbf{1}} \backslash\left\{\cup_{\{1, \beta\} \subseteq[m]} f_{1\{1, \beta\}}\right\}, \mathbf{f}_{\mathbf{2}} \backslash\left\{\cup_{\{2, \beta\} \subseteq[m]} f_{2\{2, \beta\}}\right\}, \ldots, \mathbf{f}_{\mathbf{m}} \backslash\left\{\cup_{\{m, \beta\} \subseteq[m]} f_{m\{m, \beta\}}\right\}, A^{\oplus} \backslash\left\{\cup_{\alpha \in[m]} A_{\alpha 1}^{\oplus}\right\} \\
& U_{\alpha^{c}}=A_{\alpha 1}^{\oplus} \quad \forall \alpha \in[m],
\end{aligned}
$$

and we can write the achievable rate $R_{C A P M}$ as

$$
\begin{aligned}
& \max _{k \in[m]}\left\{H\left(\mathbf{f}_{\mathbf{1}} \backslash\left\{\cup_{\{1, \beta\} \subseteq[m]} f_{1\{1, \beta\}}\right\}, \mathbf{f}_{\mathbf{2}} \backslash\left\{\cup_{\{2, \beta\} \subseteq[m]} f_{2\{2, \beta\}}\right\}, \ldots, \mathbf{f}_{\mathbf{m}} \backslash\left\{\cup_{\{m, \beta\} \subseteq[m]} f_{m\{m, \beta\}}\right\}, A^{\oplus} \backslash\left\{\cup_{\alpha \in[m]} A_{\alpha 1}^{\oplus}\right\} \mid \mathbf{Y}_{\mathbf{k}}\right)\right\} \\
& \quad+\max _{k \in\{1\}^{c}}\left\{H\left(A_{11}^{\oplus} \mid \mathbf{Y}_{\mathbf{k}}\right)\right\}+\cdots+\max _{k \in\{m\}^{c}}\left\{H\left(A_{m 1}^{\oplus} \mid \mathbf{Y}_{\mathbf{k}}\right)\right\} .
\end{aligned}
$$

Note that

$$
\begin{aligned}
& H\left(A_{\alpha 1},\left(A_{\alpha 2}\right)_{\left|A_{\alpha 1}\right|}, \ldots,\left(A_{\alpha(m-1)}\right)_{\left|A_{\alpha 1}\right|} \mid \mathbf{Y}_{\mathbf{k}}\right) \\
& \stackrel{a}{=} H\left(A_{\alpha 1} \oplus\left(A_{\alpha 2}\right)_{\left|A_{\alpha 1}\right|} \oplus, \cdots, \oplus\left(A_{\alpha(m-1)}\right)_{\left|A_{\alpha 1}\right|} \mid \mathbf{Y}_{\mathbf{k}}\right) \\
& =H\left(A_{\alpha 1}^{\oplus} \mid \mathbf{Y}_{\mathbf{k}}\right) .
\end{aligned}
$$

Before rearranging the terms in $R_{C A P M}$ further, we would like to make the following remarks.

Remark 13. From the Definition 9. we know that for all $[m] \backslash\{\alpha\}$ the conditional entropy

$$
\begin{aligned}
& H\left(A_{\alpha 1}^{\oplus} \mid \mathbf{Y}_{\mathbf{k}}\right) \\
& =H\left(\left(A_{\alpha j}\right)_{\left|A_{\alpha 1}\right|} \mid \mathbf{Y}_{\mathbf{k}}\right) \\
& =H\left(\left(A_{\alpha j}\right)_{\left|A_{\alpha 1}\right|}\right)=\left|A_{\alpha 1}\right|=\min _{l \in[m-1]}\left|A_{\alpha l}\right| \text { bits, }
\end{aligned}
$$

where $A_{\alpha j}$ is the demand at Decoder $k$ related to $G_{m-2}$.

Hence we get,

$$
\begin{aligned}
H\left(U_{\alpha^{c}} \mid \mathbf{Y}_{\mathbf{k}}\right) & =H\left(U_{\alpha^{c}} \mid \mathbf{Y}_{\mathbf{j}}\right) \\
& =\min _{l \in[m-1]}\left|A_{\alpha l}\right| \text { bits, } \forall k, j \in\{\alpha\}^{c}
\end{aligned}
$$

Remark 14. By Definition 9. for all $\alpha \in[m]$,

$$
\begin{aligned}
H\left(A^{\oplus} \backslash A_{\alpha 1}^{\oplus} \mid \mathbf{Y}_{\alpha}\right) & =H\left(A^{\oplus} \backslash A_{\alpha}^{\oplus},\left(A_{\alpha 2}^{\oplus}, \ldots, A_{\alpha(m-1)}^{\oplus}\right) \mid \mathbf{Y}_{\alpha}\right) \\
& =H\left(A^{\oplus} \backslash A_{\alpha}^{\oplus} \mid \mathbf{Y}_{\alpha}\right)+H\left(A_{\alpha 2}^{\oplus}, \ldots, A_{\alpha(m-1)}^{\oplus} \mid \mathbf{Y}_{\alpha}\right) \\
& =H\left(\cup_{\{\alpha, \beta\} \subseteq[m]} f_{\alpha\{\alpha, \beta\}} \mid \mathbf{Y}_{\alpha}\right)+H\left(A_{\alpha 2}^{\oplus}, \ldots, A_{\alpha(m-1)}^{\oplus}\right) \\
& =H\left(\cup_{\{\alpha, \beta\} \subseteq[m]} f_{\alpha\{\alpha, \beta\}} \mid \mathbf{Y}_{\alpha}\right)+\left(\max _{j}\left|A_{\alpha j}\right|-\min _{j}\left|A_{\alpha j}\right|\right) .
\end{aligned}
$$


By Remark 13, $H\left(U_{\alpha^{c}} \mid \mathbf{Y}_{\mathbf{k}}\right)=H\left(U_{\alpha^{c}} \mid \mathbf{Y}_{\mathbf{j}}\right)$ for all $k, j \in\{\alpha\}^{c}$. When we expand the terms inside $\max _{k \in[m]}$, we can write

$$
\begin{aligned}
R_{C A P M}=\max & \left\{H\left(\mathbf{f}_{\mathbf{1}} \backslash\left\{\cup_{\{1, \beta\} \subseteq[m]} f_{1\{1, \beta\}}\right\}, \ldots, \mathbf{f}_{\mathbf{m}} \backslash\left\{\cup_{\{m, \beta\} \subseteq[m]} f_{m\{m, \beta\}}\right\}, A^{\oplus} \backslash\left\{\cup_{\alpha \in[m]} A_{\alpha 1}^{\oplus}\right\} \mid \mathbf{Y}_{\mathbf{1}}\right)\right. \\
& +\sum_{\alpha \in\{1\}^{c}} H\left(U_{\alpha^{c}} \mid \mathbf{Y}_{\mathbf{1}}\right)+\max _{k \in\{1\}^{c}}\left\{H\left(U_{1^{c}} \mid \mathbf{Y}_{\mathbf{k}}\right)\right\}, \\
& H\left(\mathbf{f}_{\mathbf{1}} \backslash\left\{\cup_{\{1, \beta\} \subseteq[m]} f_{1\{1, \beta\}}\right\}, \ldots, \mathbf{f}_{\mathbf{m}} \backslash\left\{\cup_{\{m, \beta\} \subseteq[m]} f_{m\{m, \beta\}}\right\}, A^{\oplus} \backslash\left\{\cup_{\alpha \in[m]} A_{\alpha 1}^{\oplus}\right\} \mid \mathbf{Y}_{\mathbf{2}}\right) \\
& +\sum_{\alpha \in\{2\}^{c}} H\left(U_{\alpha^{c}} \mid \mathbf{Y}_{\mathbf{2}}\right)+\max _{k \in\{2\}^{c}}\left\{H\left(U_{2^{c}} \mid \mathbf{Y}_{\mathbf{k}}\right)\right\} \\
& \ldots, \\
& H\left(\mathbf{f}_{\mathbf{1}} \backslash\left\{\cup_{\{1, \beta\} \subseteq[m]} f_{1\{1, \beta\}}\right\}, \ldots, \mathbf{f}_{\mathbf{m}} \backslash\left\{\cup_{\{m, \beta\} \subseteq[m]} f_{m\{m, \beta\}}\right\}, A^{\oplus} \backslash\left\{\cup_{\alpha \in[m]} A_{\alpha 1}^{\oplus}\right\} \mid \mathbf{Y}_{\mathbf{m}}\right) \\
& \left.+\sum_{\alpha \in\{m\}^{c}} H\left(U_{\alpha^{c}} \mid \mathbf{Y}_{\mathbf{m}}\right)+\max _{k \in\{m\}^{c}}\left\{H\left(U_{m^{c}} \mid \mathbf{Y}_{\mathbf{k}}\right)\right\}\right\} .
\end{aligned}
$$

Since $H\left(U_{\alpha^{c}} \mid \mathbf{Y}_{\mathbf{k}}\right)=H\left(A_{\alpha 1}^{\oplus} \mid \mathbf{Y}_{\mathbf{k}}\right)$ and $H\left(U_{\alpha^{c}} \mid \mathbf{Y}_{\mathbf{k}}\right)=\left|A_{\alpha 1}^{\oplus}\right|$ for all $k \in\{\alpha\}^{c}$ by Remark 13 , we can further write $R_{C A P M}$ as

$$
\begin{aligned}
\max & \left\{H\left(\mathbf{f}_{\mathbf{1}} \backslash\left\{\cup_{\{1, \beta\} \subseteq[m]} f_{1\{1, \beta\}}\right\}, \ldots, \mathbf{f}_{\mathbf{m}} \backslash\left\{\cup_{\{m, \beta\} \subseteq[m]} f_{m\{m, \beta\}}\right\}, A^{\oplus} \backslash\left\{\cup_{\alpha \in[m]} A_{\alpha 1}^{\oplus}\right\} \mid \mathbf{Y}_{\mathbf{1}}\right)\right. \\
& +\sum_{\alpha \in\{1\}^{c}} H\left(A_{\alpha 1}^{\oplus} \mid \mathbf{Y}_{\mathbf{1}}\right)+\left|A_{11}^{\oplus}\right|, \\
& H\left(\mathbf{f}_{\mathbf{1}} \backslash\left\{\cup_{\{1, \beta\} \subseteq[m]} f_{1\{1, \beta\}}\right\}, \ldots, \mathbf{f}_{\mathbf{m}} \backslash\left\{\cup_{\{m, \beta\} \subseteq[m]} f_{m\{m, \beta\}}\right\}, A^{\oplus} \backslash\left\{\cup_{\alpha \in[m]} A_{\alpha 1}^{\oplus}\right\} \mid \mathbf{Y}_{\mathbf{2}}\right) \\
& +\sum_{\alpha \in\{2\}^{c}} H\left(A_{\alpha 1}^{\oplus} \mid \mathbf{Y}_{\mathbf{2}}\right)+\left|A_{21}^{\oplus}\right| \\
& , \ldots, \\
& H\left(\mathbf{f}_{\mathbf{1}} \backslash\left\{\cup_{\{1, \beta\} \subseteq[m]} f_{1\{1, \beta\}}\right\}, \ldots, \mathbf{f}_{\mathbf{m}} \backslash\left\{\cup_{\{m, \beta\} \subseteq[m]} f_{m\{m, \beta\}}\right\}, A^{\oplus} \backslash\left\{\cup_{\alpha \in[m]} A_{\alpha 1}^{\oplus}\right\} \mid \mathbf{Y}_{\mathbf{m}}\right) \\
& \left.+\sum_{\alpha \in\{m\}^{c}} H\left(A_{\alpha 1}^{\oplus} \mid \mathbf{Y}_{\mathbf{m}}\right)+\left|A_{m 1}^{\oplus}\right|\right\} .
\end{aligned}
$$

By applying chain rule to the expression above and since all $U_{i^{c}}=A_{i 1}^{\oplus}$ satisfy the condition 2 in Proposition 1 , we have

$$
\begin{aligned}
& R_{C A P M}=\max \left\{H\left(\mathbf{f}_{\mathbf{1}} \backslash\left\{\cup_{\{1, \beta\} \subseteq[m]} f_{1\{1, \beta\}}\right\}, \ldots, \mathbf{f}_{\mathbf{m}} \backslash\left\{\cup_{\{m, \beta\} \subseteq[m]} f_{m\{m, \beta\}}\right\}, A^{\oplus} \backslash\left\{\cup_{\alpha \in[m]} A_{\alpha 1}^{\oplus}\right\} \mid \mathbf{Y}_{\mathbf{1}}\right)\right. \\
& +H\left(\cup_{\alpha \in\{1\}} A_{\alpha 1}^{\oplus} \mid \mathbf{Y}_{\mathbf{1}}\right)+\left|A_{11}^{\oplus}\right|, \\
& H\left(\mathbf{f}_{\mathbf{1}} \backslash\left\{\cup_{\{1, \beta\} \subseteq[m]} f_{1\{1, \beta\}}\right\}, \ldots, \mathbf{f}_{\mathbf{m}} \backslash\left\{\cup_{\{m, \beta\} \subseteq[m]} f_{m\{m, \beta\}}\right\}, A^{\oplus} \backslash\left\{\cup_{\alpha \in[m]} A_{\alpha 1}^{\oplus}\right\} \mid \mathbf{Y}_{\mathbf{2}}\right) \\
& +H\left(\cup_{\alpha \in\{2\}^{c}} A_{\alpha 1}^{\oplus} \mid \mathbf{Y}_{2}\right)+\left|A_{21}^{\oplus}\right| \\
& , \ldots \text {, } \\
& H\left(\mathbf{f}_{\mathbf{1}} \backslash\left\{\cup_{\{1, \beta\} \subseteq[m]} f_{1\{1, \beta\}}\right\}, \ldots, \mathbf{f}_{\mathbf{m}} \backslash\left\{\cup_{\{m, \beta\} \subseteq[m]} f_{m\{m, \beta\}}\right\}, A^{\oplus} \backslash\left\{\cup_{\alpha \in[m]} A_{\alpha 1}^{\oplus}\right\} \mid \mathbf{Y}_{\mathbf{m}}\right) \\
& \left.+H\left(\cup_{\alpha \in\{m\}^{c}} A_{\alpha 1}^{\oplus} \mid \mathbf{Y}_{\mathbf{m}}\right)+\left|A_{m 1}^{\oplus}\right|\right\} .
\end{aligned}
$$

Applying chain rule once more and since all $U_{i^{c}}=A_{i 1}^{\oplus}$ satisfy the condition 2 in Proposition 1, we get

$$
\begin{aligned}
R_{C A P M}=\max & \left\{H\left(\mathbf{f}_{\mathbf{1}} \backslash\left\{\cup_{\{1, \beta\} \subseteq[m]} f_{1\{1, \beta\}}\right\}, \ldots, \mathbf{f}_{\mathbf{m}} \backslash\left\{\cup_{\{m, \beta\} \subseteq[m]} f_{m\{m, \beta\}}\right\} \mid \mathbf{Y}_{\mathbf{1}}\right)+H\left(A^{\oplus} \backslash A_{11}^{\oplus} \mid \mathbf{Y}_{\mathbf{1}}\right)+\left|A_{11}^{\oplus}\right|,\right. \\
& H\left(\mathbf{f}_{\mathbf{1}} \backslash\left\{\cup_{\{1, \beta\} \subseteq[m]} f_{1\{1, \beta\}}\right\}, \ldots, \mathbf{f}_{\mathbf{m}} \backslash\left\{\cup_{\{m, \beta\} \subseteq[m]} f_{m\{m, \beta\}}\right\} \mid \mathbf{Y}_{\mathbf{2}}\right)+H\left(A^{\oplus} \backslash A_{21}^{\oplus} \mid \mathbf{Y}_{\mathbf{2}}\right)+\left|A_{21}^{\oplus}\right| \\
& , \ldots, \\
& \left.H\left(\mathbf{f}_{\mathbf{1}} \backslash\left\{\cup_{\{1, \beta\} \subseteq[m]} f_{1\{1, \beta\}}\right\}, \ldots, \mathbf{f}_{\mathbf{m}} \backslash\left\{\cup_{\{m, \beta\} \subseteq[m]} f_{m\{m, \beta\}}\right\} \mid \mathbf{Y}_{\mathbf{m}}\right)+H\left(A^{\oplus} \backslash A_{m 1}^{\oplus} \mid \mathbf{Y}_{\mathbf{m}}\right)+\left|A_{m 1}^{\oplus}\right|\right\} .
\end{aligned}
$$

Finally, using Remark 14 that $\left|A_{\alpha 1}\right|=\min _{j}\left|A_{\alpha j}\right|$ and chain rule, we have the following expression for $R_{C A P M}$.

$$
\begin{aligned}
R_{C A P M}= & \max \left\{H\left(\mathbf{f}_{\mathbf{1}}, \mathbf{f}_{\mathbf{2}} \backslash\left\{\cup_{\{2, \beta\} \subseteq[m]} f_{2\{2, \beta\}}\right\}, \ldots, \mathbf{f}_{\mathbf{m}} \backslash\left\{\cup_{\{m, \beta\} \subseteq[m]} f_{m\{m, \beta\}}\right\} \mid \mathbf{Y}_{\mathbf{1}}\right)+\max _{j}\left|A_{1 j}\right|,\right. \\
& H\left(\mathbf{f}_{\mathbf{1}} \backslash\left\{\cup_{\{1, \beta\} \subseteq[m]} f_{1\{1, \beta\}}\right\}, \mathbf{f}_{\mathbf{2}}, \mathbf{f}_{\mathbf{3}} \backslash\left\{\cup_{\{3, \beta\} \subseteq[m]} f_{3\{3, \beta\}}\right\}, \ldots, \mathbf{f}_{\mathbf{m}} \backslash\left\{\cup_{\{m, \beta\} \subseteq[m]} f_{m\{m, \beta\}}\right\} \mid \mathbf{Y}_{\mathbf{2}}\right)+\max _{j}\left|A_{2 j}\right| \\
& , \ldots, \\
& \left.H\left(\mathbf{f}_{\mathbf{1}} \backslash\left\{\cup_{\{1, \beta\} \subseteq[m]} f_{1\{1, \beta\}}\right\}, \ldots, \mathbf{f}_{\mathbf{m}-\mathbf{1}} \backslash\left\{\cup_{\{m-1, \beta\} \subseteq[m]} f_{m-1\{m-1, \beta\}}\right\}, \mathbf{f}_{\mathbf{m}} \mid \mathbf{Y}_{\mathbf{m}}\right)+\max _{j}\left|A_{m j}\right|\right\} .
\end{aligned}
$$


Then we can write this achievable rate for the problem, $R_{C A P M}$ in 29] as

$$
R_{C A P M}=\max \left\{R_{1}, \ldots, R_{m}\right\}
$$

Next, we find a lower bound which matches $R_{C A P M}$ above by utilizing the converse result in section $\mathrm{V}$. Let us focus on (19). Here, we can write

$$
\begin{aligned}
& H\left(\mathbf{f}_{\mathbf{2}} \mid \mathbf{Y}_{\mathbf{2}}, \mathbf{f}_{\mathbf{1}}, \mathbf{Y}_{\mathbf{1}}\right) \\
& =H\left(\mathbf{f}_{\mathbf{2}} \mid \mathbf{f}_{\mathbf{1}}, \mathbf{Y}_{\mathbf{1}}\right) \\
& =H\left(\mathbf{f}_{\mathbf{2}} \backslash\left\{\cup_{\{2, \beta\} \in[m]} f_{2\{2, \beta\}}\right\},\left\{\cup_{\{2, \beta\} \in[m]} f_{2\{2, \beta\}}\right\} \mid \mathbf{f}_{\mathbf{1}}, \mathbf{Y}_{\mathbf{1}}\right) \\
& \left.=H\left(\mathbf{f}_{\mathbf{2}} \backslash\left\{\cup_{\{2, \beta\} \in[m]} f_{2\{2, \beta\}}\right\}, f_{2\{2,1\}}\right\} \mid \mathbf{f}_{\mathbf{1}}, \mathbf{Y}_{\mathbf{1}}\right) \\
& =H\left(\mathbf{f}_{\mathbf{2}} \backslash\left\{\cup_{\{2, \beta\} \in[m]} f_{2\{2, \beta\}}\right\}, A_{1 j} \mid \mathbf{f}_{\mathbf{1}}, \mathbf{Y}_{\mathbf{1}}\right), \\
& \text { where } A_{1 j}=f_{2\{2,1\}} \\
& =H\left(\mathbf{f}_{\mathbf{2}} \backslash\left\{\cup_{\{2, \beta\} \in[m]} f_{2\{2, \beta\}}\right\} \mid \mathbf{f}_{\mathbf{1}}, \mathbf{Y}_{\mathbf{1}}\right)+H\left(A_{1 j}\right) \\
& =H\left(\mathbf{f}_{\mathbf{2}} \backslash\left\{\cup_{\{2, \beta\} \in[m]} f_{2\{2, \beta\}}\right\} \mid \mathbf{f}_{\mathbf{1}}, \mathbf{Y}_{\mathbf{1}}\right)+\left|A_{1 j}\right|
\end{aligned}
$$

and

$$
\begin{aligned}
& H\left(\mathbf{f}_{\mathbf{3}} \mid \mathbf{Y}_{\mathbf{3}}, \mathbf{f}_{\mathbf{2}}, \mathbf{Y}_{\mathbf{2}}, \mathbf{f}_{\mathbf{1}}, \mathbf{Y}_{\mathbf{1}}\right) \\
& =H\left(\mathbf{f}_{\mathbf{3}} \mid \mathbf{f}_{\mathbf{2}}, \mathbf{Y}_{\mathbf{2}}, \mathbf{f}_{\mathbf{1}}, \mathbf{Y}_{\mathbf{1}}\right) \\
& =H\left(\mathbf{f}_{\mathbf{3}} \backslash\left\{\cup_{\{3, \beta\} \in[m]} f_{3\{3, \beta\}}\right\},\left\{\cup_{\{3, \beta\} \in[m]} f_{3\{3, \beta\}}\right\} \mid \mathbf{f}_{\mathbf{2}}, \mathbf{Y}_{\mathbf{2}}, \mathbf{f}_{\mathbf{1}}, \mathbf{Y}_{\mathbf{1}}\right) \\
& =H\left(\mathbf{f}_{\mathbf{3}} \backslash\left\{\cup_{\{3, \beta\} \in[m]} f_{3\{3, \beta\}}\right\} \mid \mathbf{f}_{\mathbf{2}}, \mathbf{Y}_{\mathbf{2}}, \mathbf{f}_{\mathbf{1}}, \mathbf{Y}_{\mathbf{1}}\right), \\
& \text { since }\left\{\cup_{\{3, \beta\} \in[m]} f_{3\{3, \beta\}}\right\} \subset\left\{\mathbf{Y}_{\mathbf{1}}, \mathbf{Y}_{\mathbf{2}}\right\} \\
& =H\left(\mathbf{f}_{\mathbf{3}} \backslash\left\{\cup_{\{3, \beta\} \in[m]} f_{3\{3, \beta\}}\right\} \mid \mathbf{f}_{\mathbf{2}} \backslash\left\{\cup_{\{2, \beta\} \in[m]} f_{2\{2, \beta\}}\right\}, \mathbf{Y}_{\mathbf{2}}, \mathbf{f}_{\mathbf{1}}, \mathbf{Y}_{\mathbf{1}}\right), \\
& \text { since }\left\{\cup_{\{2, \beta\} \in[m]} f_{2\{2, \beta\}}\right\} \perp \mathbf{f}_{\mathbf{3}} \backslash\left\{\cup_{\{3, \beta\} \in[m]} f_{3\{3, \beta\}}\right\} \mid \mathbf{Y}_{\mathbf{i}}, \mathbf{f}_{\mathbf{j}} \text { for all } i, j \in[m] .
\end{aligned}
$$

Note that $\mathbf{f}_{\mathbf{3}}$ can be written as

$$
\left\{f_{3[m]}, f_{33}, \cup_{\{3, \beta\} \in[m]} f_{3\{3, \beta\}}, \cup_{\{3, \beta\} \in[m]} f_{\{3, \beta\}\{3, \beta\}}\right\} .
$$

Then we get the following equality:

$$
\mathbf{f}_{\mathbf{3}} \backslash\left\{\cup_{\{3, \beta\} \in[m]} f_{3\{3, \beta\}}\right\}=\left\{f_{3[m]}, f_{33}, \cup_{\{3, \beta\} \in[m]} f_{\{3, \beta\}\{3, \beta\}}\right\} .
$$

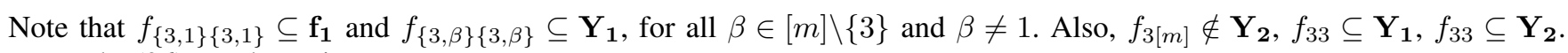
As a result, 36 can be written as

$$
H\left(\mathbf{f}_{\mathbf{3}} \backslash\left\{\cup_{\{3, \beta\} \in[m]} f_{3\{3, \beta\}}\right\} \mid \mathbf{f}_{\mathbf{2}} \backslash\left\{\cup_{\{2, \beta\} \in[m]} f_{2\{2, \beta\}}\right\}, \mathbf{f}_{\mathbf{1}}, \mathbf{Y}_{\mathbf{1}}\right) .
$$

By similar arguments as above, for $p>2$ we can write

$$
\begin{aligned}
& H\left(\mathbf{f}_{\mathbf{p}} \mid \mathbf{Y}_{\mathbf{p}}, \mathbf{f}_{\mathbf{p}-\mathbf{1}}, \mathbf{Y}_{\mathbf{p}-\mathbf{1}}, \ldots, \mathbf{f}_{\mathbf{1}}, \mathbf{Y}_{\mathbf{1}}\right) \\
& =H\left(\mathbf{f}_{\mathbf{p}} \mid \mathbf{f}_{\mathbf{p}-\mathbf{1}}, \mathbf{Y}_{\mathbf{p}-\mathbf{1}}, \ldots, \mathbf{f}_{\mathbf{1}}, \mathbf{Y}_{\mathbf{1}}\right) \\
& =H\left(\mathbf{f}_{\mathbf{p}} \backslash\left\{\cup_{\{p, \beta\} \in[m]} f_{p\{p, \beta\}}\right\}, \cup_{\{p, \beta\} \in[m]} f_{p\{p, \beta\}} \mid \mathbf{f}_{\mathbf{p}-\mathbf{1}}, \mathbf{Y}_{\mathbf{p}-\mathbf{1}}, \ldots, \mathbf{f}_{\mathbf{1}}, \mathbf{Y}_{\mathbf{1}}\right) \\
& \stackrel{a}{=} H\left(\mathbf{f}_{\mathbf{p}} \backslash\left\{\cup_{\{p, \beta\} \in[m]} f_{p\{p, \beta\}}\right\} \mid \mathbf{f}_{\mathbf{p}-\mathbf{1}}, \mathbf{Y}_{\mathbf{p}-\mathbf{1}}, \ldots, \mathbf{f}_{\mathbf{1}}, \mathbf{Y}_{\mathbf{1}}\right) \\
& \stackrel{b}{=} H\left(\mathbf{f}_{\mathbf{p}} \backslash\left\{\cup_{\{p, \beta\} \in[m]} f_{p\{p, \beta\}}\right\} \mid \mathbf{f}_{\mathbf{p}-\mathbf{1}} \backslash\left\{\cup_{\{p-1, \beta\} \in[m]} f_{p-1\{p-1, \beta\}}\right\}, \mathbf{Y}_{\mathbf{p}-\mathbf{1}}, \ldots, \mathbf{f}_{\mathbf{2}} \backslash\left\{\cup_{\{2, \beta\} \in[m]} f_{2\{2, \beta\}}\right\}, \mathbf{Y}_{\mathbf{2}}, \mathbf{f}_{\mathbf{1}}, \mathbf{Y}_{\mathbf{1}}\right) \\
& \stackrel{c}{=} H\left(\mathbf{f}_{\mathbf{p}} \backslash\left\{\cup_{\{p, \beta\} \in[m]} f_{p\{p, \beta\}}\right\} \mid \mathbf{f}_{\mathbf{p}-\mathbf{1}} \backslash\left\{\cup_{\{p-1, \beta\} \in[m]} f_{p-1\{p-1, \beta\}}\right\}, \ldots, \mathbf{f}_{\mathbf{2}} \backslash\left\{\cup_{\{2, \beta\} \in[m]} f_{2\{2, \beta\}}\right\}, \mathbf{f}_{\mathbf{1}}, \mathbf{Y}_{\mathbf{1}}\right)
\end{aligned}
$$

a: Since $\left\{\cup_{\{p, \beta\} \in[m]} f_{p\{p, \beta\}}\right\} \subset\left\{\mathbf{Y}_{\mathbf{i}}, \mathbf{Y}_{\mathbf{j}}\right\}, \forall i, j \in[m], p \neq i, j$ and $i \neq j$.

b: Since $\left\{\cup_{\{\alpha, \beta\} \in[m]} f_{\alpha\{\alpha, \beta\}}\right\} \perp \mathbf{f}_{\gamma} \backslash\left\{\cup_{\{\gamma, \beta\} \in[m]} f_{\gamma\{\gamma, \beta\}}\right\} \quad \mid \mathbf{Y}_{\mathbf{i}}, \mathbf{f}_{\mathbf{j}}, \forall i, j, \alpha, \gamma \in[m]$.

c: Since $\mathbf{f}_{\mathbf{p}}=\left\{f_{p[m]}, f_{p p},\left\{\cup_{\{p, \beta\} \in[m]} f_{p\{p, \beta\}}\right\},\left\{\cup_{\{p, \beta\} \in[m]} f_{\{p, \beta\}\{p, \beta\}}\right\},\right\}$ and $\mathbf{f}_{\mathbf{p}} \backslash\left\{\cup_{\{p, \beta\} \in[m]} f_{p\{p, \beta\}}\right\}$ equals $\left\{f_{p[m]}, f_{p p},\left\{\cup_{\{p, \beta\} \in[m]} f_{\{p, \beta\}\{p, \beta\}}\right\}\right\}$, where $f_{p[m]} \notin \mathbf{Y}_{\mathbf{i}}, \forall i \in[m]$ and $f_{p p} \subseteq \mathbf{Y}_{\mathbf{i}} \forall i \in[m], i \neq q$. Also, $f_{\{p, 1\}\{p, 1\}} \subseteq \mathbf{f}_{\mathbf{1}}$ and $f_{\{p, \beta\}\{p, \beta\}} \subseteq \mathbf{Y}_{\mathbf{1}}, \forall \beta \in[m] \backslash\{p\}, \beta \neq 1$.

Hence, from (34) and (37), (19) can be written as

$$
R \geq H\left(\mathbf{f}_{\mathbf{1}}, \mathbf{f}_{\mathbf{2}} \backslash\left\{\cup_{\{2, \beta\} \in[m]} f_{2\{2, \beta\}}\right\}, \ldots, \mathbf{f}_{\mathbf{m}} \backslash\left\{\cup_{\{m, \beta\} \in[m]} f_{m\{m, \beta\}}\right\} \mid \mathbf{Y}_{\mathbf{1}}\right)+\left|A_{1 j}\right|,
$$

where $A_{1 j}=f_{2\{2,1\}}$. 
By similar arguments used to obtain 38 , we can write

$$
R \geq H\left(\mathbf{f}_{\mathbf{1}}, \mathbf{f}_{\mathbf{2}} \backslash\left\{\cup_{\{2, \beta\} \in[m]} f_{2\{2, \beta\}}\right\}, \ldots, \mathbf{f}_{\mathbf{m}} \backslash\left\{\cup_{\{m, \beta\} \in[m]} f_{m\{m, \beta\}}\right\} \mid \mathbf{Y}_{\mathbf{1}}\right)+\max _{j}\left|A_{1 j}\right| .
$$

Note that the right hand side of $[39)$ is $R_{1}$. Since the problem is symmetric, similarly we can get all $R_{i}$ 's. Then,

$$
R \geq \max _{i} R_{i}=R_{C A P M}
$$

proving that $R_{C A P M}$ is optimal. This concludes the proof of the Theorem 3 .

The following result illustrates the importance of excess bits.

Proposition 3. If the demands of the m-user index coding problem are such that there are no excess bits after Step 1 of CAPM then the rate obtained by following only Step 1 is optimal. The optimal rate $R_{*}$ can be written as

$$
\begin{aligned}
R_{*}= & \max _{\pi}\left\{H\left(\mathbf{f}_{\pi(\mathbf{1})} \mid \mathbf{Y}_{\pi(\mathbf{1})}\right)+H\left(\mathbf{f}_{\pi(\mathbf{2})} \mid \mathbf{f}_{\pi(\mathbf{1})}, \mathbf{Y}_{\pi(\mathbf{1})}, \mathbf{Y}_{\pi(\mathbf{2})}\right)\right. \\
& +\cdots+ \\
& \left.H\left(\mathbf{f}_{\pi(\mathbf{m})} \mid \mathbf{f}_{\pi(\mathbf{1})}, \mathbf{Y}_{\pi(\mathbf{1})}, \ldots, \mathbf{f}_{\pi(\mathbf{m}-\mathbf{1})}, \mathbf{Y}_{\pi(\mathbf{m}-\mathbf{1})}, \mathbf{Y}_{\pi(\mathbf{m})}\right)\right\}
\end{aligned}
$$

where $\pi($.$) denotes the following m$ permutations on $[\mathrm{m}]$ :

$$
(1,2, \ldots, m),(2,1,3, \ldots, m), \ldots,(m, 1, \ldots, m-1)
$$

Proof:

First we show that the achievable rate we get by applying Step 1 of CAPM gives the expression in (41). We begin with the following three observations. Firstly, all demands of each Decoder $i, \mathbf{f}_{\mathbf{i}}$, are in $\cup_{i \in I} U_{I}$. Secondly, since the demands are such that there are no excess bits after Step $1, H\left(U_{I} \mid \mathbf{Y}_{\mathbf{i}}\right)=H\left(U_{I} \mid \mathbf{Y}_{\mathbf{j}}\right)$, for all $i, j \in I \subset[m]$. Lastly, demands placed in $U_{[m]}$ at Step 1 cannot be excess bits since $U_{[m]}$ is the highest level message. Hence $H\left(U_{[m]} \mid \mathbf{Y}_{\mathbf{i}}\right)$ does not have to be equal for all $i \in[m]$.

We can write the achievable rate $R_{\mathrm{CAPM}}$ as

$$
\begin{gathered}
R_{\mathrm{CAPM}}=\max \left\{R_{1}, \ldots, R_{m}\right\}, \text { where } \\
R_{i}=H\left(U_{[m]} \mid \mathbf{Y}_{\mathbf{i}}\right)+\sum_{I \subset[m]} \max _{j \in I}\left\{H\left(U_{I} \mid \mathbf{Y}_{\mathbf{j}}\right)\right\} \\
\stackrel{a}{=} H\left(U_{[m]} \mid \mathbf{Y}_{\mathbf{i}}\right)+\sum_{I \subset[m]} H\left(U_{I} \mid \mathbf{Y}_{\mathbf{i}_{\mathbf{I}}}\right),
\end{gathered}
$$

where $i_{I}$ is an arbitrary element of $I$ and $a$ is due to the assumption that $H\left(U_{I} \mid \mathbf{Y}_{\mathbf{i}}\right)=H\left(U_{I} \mid \mathbf{Y}_{\mathbf{j}}\right)$, for all $i, j \in I \subset[m]$.

Let us focus on $R_{1}$. From (42), we can write $R_{1}$ as

$$
R_{1}=\sum_{C_{1}} H\left(U_{I} \mid \mathbf{Y}_{\mathbf{1}}\right)+\sum_{C_{2}} H\left(U_{I} \mid \mathbf{Y}_{\mathbf{2}}\right)+\cdots+\sum_{C_{m}} H\left(U_{I} \mid \mathbf{Y}_{\mathbf{m}}\right)
$$

where $C_{1}=\{I \subseteq[m] \mid 1 \in I\}, C_{2}=\{I \subseteq[m] \mid 2 \in I, 1 \notin I\}, \ldots, C_{m}=\{I \subseteq[m] \mid m \in I, 1 \notin I, \ldots, m-1 \notin I\}$.

Since all $U_{I}$ 's satisfy the condition 2) in Proposition 1, $R_{1}$ equals

$$
\begin{aligned}
& H\left(\cup_{C_{1}} U_{I} \mid \mathbf{Y}_{\mathbf{1}}\right)+H\left(\cup_{C_{2}} U_{I} \mid \mathbf{Y}_{\mathbf{2}}\right)+\cdots+H\left(\cup_{C_{m}} U_{I} \mid \mathbf{Y}_{\mathbf{m}}\right) \\
& =H\left(\mathbf{U}_{\mathbf{1}} \mid \mathbf{Y}_{\mathbf{1}}\right)+H\left(\mathbf{U}_{\mathbf{2}} \backslash \mathbf{U}_{\mathbf{1}} \mid \mathbf{Y}_{\mathbf{2}}\right)+\cdots+H\left(\mathbf{U}_{\mathbf{m}} \backslash\left\{\mathbf{U}_{\mathbf{1}}, \ldots, \mathbf{U}_{\mathbf{m}-\mathbf{1}}\right\} \mid \mathbf{Y}_{\mathbf{m}}\right),
\end{aligned}
$$

where $\mathbf{U}_{\mathbf{i}}$ is defined as $\cup_{I \subseteq[m]: i \in I} U_{I}$. By Step 1, no decoder in $I^{c}$ can demand any source bit in $U_{I}$ or have it as side information. Then we can write $R_{1}$ as

$$
R_{1}=H\left(\mathbf{U}_{\mathbf{1}} \mid \mathbf{Y}_{\mathbf{1}}\right)+H\left(\mathbf{U}_{\mathbf{2}} \backslash \mathbf{U}_{\mathbf{1}} \mid \mathbf{Y}_{\mathbf{1}}, \mathbf{Y}_{\mathbf{2}}\right)+\cdots+H\left(\mathbf{U}_{\mathbf{m}} \backslash\left\{\mathbf{U}_{\mathbf{1}}, \ldots, \mathbf{U}_{\mathbf{m}-\mathbf{1}}\right\} \mid \mathbf{Y}_{\mathbf{1}}, \ldots, \mathbf{Y}_{\mathbf{m}}\right) .
$$

Also, by Step 1 , each $U_{I}$ consists of those source bits such that, for each decoder $i$ in $I$, Decoder $i$ either demands the bit or has it as side information. Then 43 becomes

$$
\begin{gathered}
H\left(\mathbf{f}_{\mathbf{1}} \mid \mathbf{Y}_{\mathbf{1}}\right)+H\left(\mathbf{f}_{\mathbf{2}} \backslash \mathbf{f}_{\mathbf{1}} \mid \mathbf{Y}_{\mathbf{1}}, \mathbf{Y}_{\mathbf{2}}\right)+\cdots+H\left(\mathbf{f}_{\mathbf{m}} \backslash\left\{\mathbf{f}_{\mathbf{1}}, \ldots, \mathbf{f}_{\mathbf{m}-\mathbf{1}}\right\} \mid \mathbf{Y}_{\mathbf{1}}, \ldots, \mathbf{Y}_{\mathbf{m}}\right) \\
=H\left(\mathbf{f}_{\mathbf{1}} \mid \mathbf{Y}_{\mathbf{1}}\right)+H\left(\mathbf{f}_{\mathbf{2}} \mid \mathbf{f}_{\mathbf{1}}, \mathbf{Y}_{\mathbf{1}}, \mathbf{Y}_{\mathbf{2}}\right)+\cdots+H\left(\mathbf{f}_{\mathbf{m}} \mid \mathbf{f}_{\mathbf{1}}, \ldots, \mathbf{f}_{\mathbf{m}-\mathbf{1}}, \mathbf{Y}_{\mathbf{1}}, \ldots, \mathbf{Y}_{\mathbf{m}}\right) .
\end{gathered}
$$

Note that the expression for $R_{1}$ in $(44)$ is equivalent to first expression of the $R_{*}$. Applying the procedure above to the other $R_{i}$ 's similarly, we see that $R_{\mathrm{CAPM}}$ gives the expression in 41 . Evidently this expression cannot exceed the lower bound in Theorem 2, so the proof is complete. 
The coded caching problem, which was introduced by Maddah-Ali and Niesen [29], is closely related to the index coding problem. The coded caching problem consists of two phases, called the cache allocation phase and the delivery phase. During the cache allocation phase, the server can decide how to populate the caches of the various users. Each user then selects some content to demand, and during the delivery phase the server must broadcast a common message to all of the clients that allows each one to meet its demand, given its cache contents. Thus the delivery phase of the coded caching problem can be viewed as an index coding problem.

If we perform the cache allocation as in [30] and each user demands a different file at the delivery phase, then the instance of the index coding problem that results during the delivery phase satisfies the conditions in Proposition 3 in a certain asymptotic sense. Therefore, CAPM gives the optimal rate for the delivery phase in this case.

\section{S-CAPM: A Heuristic Achieving Fractional Rates}

Recall that CAPM can only give integer rates. However, some instances of the index coding problem are known to have non-integer optimal rates. We next show how CAPM can be modified to give nonintegral rate bounds, and this modification performs strictly better than CAPM in some examples. The extension is not polynomial-time computable, however. The following multi-letter extension of Proposition 4 is necessary.

Proposition 4. Let $t$ be a positive integer. The optimal rate $R_{\text {opt }}$ of an index coding problem is upper bounded by

$$
\min \frac{1}{t} \sum_{I \subseteq[m]}\left[\max _{i \in I} H\left(U_{I}^{t} \mid \mathbf{Y}_{\mathbf{i}}^{\mathbf{t}}\right)\right]
$$

where the minimization is over the set of all random variables $U_{I}^{t}$ jointly distributed with $\mathbf{S}^{\mathbf{t}}$ such that 1) There exist functions

$g_{1}\left(\cup_{1 \in I} U_{I}^{t}, \mathbf{Y}_{1}^{\mathbf{t}}\right), \ldots, g_{m}\left(\cup_{m \in I} U_{I}^{t}, \mathbf{Y}_{\mathbf{m}}^{\mathbf{t}}\right)$ such that

$$
g_{i}\left(\cup_{i \in I} U_{I}^{t}, \mathbf{Y}_{\mathbf{i}}^{\mathbf{t}}\right)=\mathbf{f}_{\mathbf{i}}^{\mathbf{t}}(\mathbf{S}), \text { for all } i \in[m]
$$

2) The auxiliary random variables $U_{I}^{t}, I \subseteq[m]$ are independent, and for all collections of subsets $J_{1}, \ldots J_{j}, K_{1}, \ldots K_{k}$, $L_{1}, \ldots, L_{l}$, and all subsets $\left\{i_{1}, \ldots, i_{p}\right\} \subseteq[\bar{m}]$, we have that $\left(U_{J_{1}}^{t}, \ldots, U_{J_{j}}^{t}\right)$ and $\left(U_{K_{1}}^{t}, \ldots, U_{K_{k}}^{t}\right)$ are conditionally independent given $\left(\left(U_{L_{1}}^{t}, \ldots, U_{L_{l}}^{t}\right),\left(\mathbf{Y}_{\mathbf{i}_{1}}^{\mathbf{t}}, \ldots, \mathbf{Y}_{\mathbf{i}_{\mathbf{p}}}^{\mathbf{t}}\right)\right.$, provided that the collections $J_{1}, \ldots J_{j}$ and $K_{1}, \ldots K_{k}$ are disjoint.

3) Each $U_{I}^{t}$ is a (possibly empty) vector of bits, each of which is the mod-2 sum of a set (possibly singleton) of source components.

Proposition 1 can evidently be recovered from Proposition 4 by taking $t=1$. But Proposition 1 actually implies Proposition 4 since the latter can be obtained by applying the former to blocks of size $t$.

Now we provide a heuristic, which we call Split Coded Approximate Partition Multicast (S-CAPM), for selecting the auxiliary random variables in Proposition 4. The steps for S-CAPM are very similar to the ones for CAPM in Section VII except for the placement of leftover bits.

Step 1 (Initialization) : This step is exactly the same as in CAPM, except that we shall parametrize the solution differently. For each $k \in\{1, \ldots,|\mathbf{S}|\}$ and each subset $I \subseteq[m]$, let $\theta(I, k)$ denote a variable in the interval $[0,1]$. We shall interpret $\theta(I, k)$ as the "fraction" of source bit $S_{k}$ that is allocated to the auxiliary random variable $U_{I}$. All such variables are initially zero.

For each source component $k$ we set $\theta\left(K \cup J^{c}, k\right)=1$, where $K$ and $J$ are chosen so that $S_{k}$ is in $f_{K J}$. This is assuming that $J \neq[m]$. As in CAPM, if $J=[m]$ then we set $\theta([m], k)=1$. Note that after this has been done for each $k$, we have

$$
\sum_{I \subset[m]} \theta(I, k)=1
$$

for each $k$. This equality will remain true after Step 2.

Step 2 : As with CAPM, the goal of Step 2 is to promote "excess bits" to a higher-level message. Since each auxiliary random variable now stores fractional bits, however, both the notion of "excess" and the promotion process are more involved.

Given the variables $\{\theta(I, k)\}$, let us define the "conditional entropy" of $U_{I}$ given $\mathbf{Y}_{\mathbf{j}}$ as

$$
H\left(U_{I} \mid \mathbf{Y}_{\mathbf{j}}\right)=\sum_{k: S_{k} \notin \mathbf{Y}_{\mathbf{j}}} \theta(I, k) .
$$

Note that if $\theta(I, k) \in\{0,1\}$ for all $I$ and $k$, then this reduces to the conditional entropy examined in Step 2 of CAPM. We shall be most interested in $H\left(U_{I} \mid \mathbf{Y}_{\mathbf{j}}\right)$ when $j \in I$, although the definition in 45 does not require this.

We then perform the following procedure for each subset $I$. The order in which we process the different subsets $I$ is not specified by the heuristic, except that if $\left|I_{1}\right|<\left|I_{2}\right|$ then $I_{1}$ must be processed prior to $I_{2}$. For a given subset $I$, we define

$$
i^{*}=\min \left\{i: H\left(U_{I} \mid \mathbf{Y}_{\mathbf{i}}\right)=\min _{l \in I} H\left(U_{I} \mid \mathbf{Y}_{\mathbf{l}}\right)\right\}
$$


and

$$
j^{*}=\min \left\{j: H\left(U_{I} \mid \mathbf{Y}_{\mathbf{j}}\right)=\max _{l \in I} H\left(U_{I} \mid \mathbf{Y}_{\mathbf{l}}\right)\right\} .
$$

If $H\left(U_{I} \mid \mathbf{Y}_{\mathbf{i}^{*}}\right)=H\left(U_{I} \mid \mathbf{Y}_{\mathbf{j}^{*}}\right)$ the we are done with this subset and may move to the next one. If $H\left(U_{I} \mid \mathbf{Y}_{\mathbf{i}^{*}}\right)<H\left(U_{I} \mid \mathbf{Y}_{\mathbf{j}^{*}}\right)$, then let $E$ denote the set of source bits that are "excess"

$$
E=\left\{k: \theta(I, k)>0 \text { and } S_{k} \in \mathbf{Y}_{\mathbf{i}^{*}} \text { but } S_{k} \notin \mathbf{Y}_{\mathbf{j}^{*}}\right\} .
$$

We then select a source bit in $E$ to promote to higher-level messages. Consider the set

$$
\left\{k \in E: \theta(I, k) \leq H\left(U_{I} \mid \mathbf{Y}_{\mathbf{j}^{*}}\right)-H\left(U_{I} \mid \mathbf{Y}_{\mathbf{i}^{*}}\right)\right\} .
$$

If this set is nonempty, then there is at least one source bit that is "entirely excess." We shall select one such bit to promote. Choose an arbitrary

$$
k^{*} \in \arg \max \left\{\theta(I, k): k \in E \text { and } \theta(I, k) \leq H\left(U_{I} \mid \mathbf{Y}_{\mathbf{j}}^{*}\right)-H\left(U_{I} \mid \mathbf{Y}_{\mathbf{i}}^{*}\right)\right\} .
$$

We then set $\theta\left(I, k^{*}\right)=0$ and we increment $\theta\left(I^{\prime}, k^{*}\right)$ for all $I^{\prime}$ such that $I \subseteq I^{\prime}$ and $\left|I^{\prime}\right|=|I|+1$ by the amount

$$
\frac{\theta\left(I, k^{*}\right)}{\left|I^{c}\right|} \text {. }
$$

In words, we view $\theta\left(I, k^{*}\right)$ as an amount of fluid that is removed from $U_{I}$ and divided equally among the $I^{c}$ sets $I^{\prime}$. If there are no bits that are entirely excess, i.e., the set in (48) is empty, then choose an arbitrary

$$
k^{*} \in \arg \min \{\theta(I, k): k \in E\} .
$$

We then promote only the portion of $\theta(I, k)$ that is excess. That is, we replace $\theta(I, k)$ with

$$
H\left(U_{I} \mid \mathbf{Y}_{\mathbf{j}}^{*}\right)-H\left(U_{I} \mid \mathbf{Y}_{\mathbf{i}}^{*}\right)
$$

and divide the remaining part,

$$
\theta(I, k)-\left(H\left(U_{I} \mid \mathbf{Y}_{\mathbf{j}}^{*}\right)-H\left(U_{I} \mid \mathbf{Y}_{\mathbf{i}}^{*}\right)\right)
$$

equally among all of the sets $I^{\prime}$ such that $I \subset I^{\prime}$ and $\left|I^{\prime}\right|=|I|+1$. Observe that since $\theta(I, k)$ must be rational for all $I$ and $k$, the process will eventually terminate.

Step 3 : As in CAPM, we now look for opportunities to exclusive-OR source bits included in the same auxiliary random variable. First we convert the fractional bits described by the $\theta(\cdot, \cdot)$ variables to an integral number by increasing the parameter $t$. Observe that $\theta(I, k)$ must be rational for each $I$ and $k$; let $t$ denote the smallest positive integer so that $\theta(I, k) \cdot t$ is an integer for all $I$ and $k$. Next recall that for each $k$

$$
\sum_{I \subseteq[m]} \theta(I, k) \cdot t=t
$$

We then divide the block of $t$ bits corresponding to source component $k$ among the $U_{I}$ variables so that the number of bits that $U_{I}$ receives is $\theta(I, k) \cdot t$. One can verify that the resulting $U_{I}$ variables satisfy conditions 1)-3) in Proposition 4 . For each $U_{I}$ variable, we then look for exclusive-OR opportunities as in Step 3 of CAPM, resulting in revised $U_{I}$ variables that remain feasible.

We next illustrate S-CAPM with two examples.

Example 4. In this case, there are 4 decoders with side information and demands as shown in Fig. 4 where $f_{\{2,3,4\} 1^{c}}=$ $\left(S_{1}, S_{2}\right), f_{21^{c}}=\left(S_{3}, S_{4}\right), f_{31^{c}}=\left(S_{5}, S_{6}\right), f_{42^{c}}=\left(S_{7}, S_{8}\right), f_{43^{c}}=\left(S_{9}, S_{10}\right), f_{\{1,2,3\} 4^{c}}=\left(S_{11}, S_{12}\right)$ and $f_{14^{c}}=S_{13}$. By using $S$-CAPM, we determine the messages and $t$ of the achievable scheme.

Step 1 : At the end of this step, all of the following $\theta(I, k)$ 's are unity:

$\theta(\{1,4\}, 13), \theta(\{1,2\}, 3), \theta(\{1,2\}, 4), \theta(\{1,3\}, 5), \theta(\{1,3\}, 6), \theta(\{2,4\}, 7), \theta(\{2,4\}, 8), \theta(\{3,4\}, 9), \theta(\{3,4\}, 10)$, $\theta([4], 1), \theta([4], 2), \theta([4], 11), \theta([4], 12)$.

Step 2: We start with level-2 messages. Note that all demands in level-2 messages are excess bits. Since there are two possible level-3 messages that each demand can move, we set all the corresponding $\theta(I, k)$ s to 0.5 . At this point the nonzero $\theta(I, k)$ 's are

$$
\begin{aligned}
& \theta(\{1,2,4\}, 13), \theta(\{1,2,4\}, 7), \theta(\{1,2,4\}, 8), \theta(\{1,2,4\}, 3), \\
& \theta(\{1,2,4\}, 4), \\
& \theta(\{1,3,4\}, 13), \theta(\{1,3,4\}, 9), \theta(\{1,3,4\}, 10), \theta(\{1,3,4\}, 5), \\
& \theta(\{1,3,4\}, 6), \\
& \theta(\{1,2,3\}, 5), \theta(\{1,2,3\}, 6), \theta(\{1,2,3\}, 3), \theta(\{1,2,3\}, 4), \\
& \theta(\{2,3,4\}, 7), \theta(\{2,3,4\}, 8), \theta(\{2,3,4\}, 9), \theta(\{2,3,4\}, 10), \\
& \theta([4], 1), \theta([4], 2), \theta([4], 11), \theta([4], 12),
\end{aligned}
$$




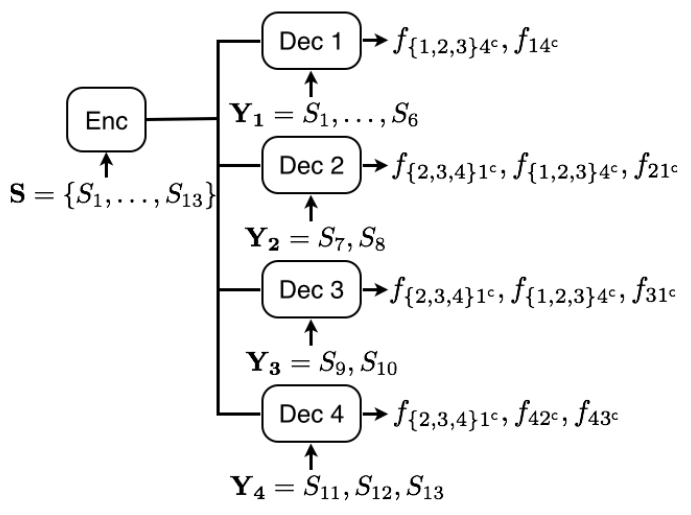

Fig. 4. Index coding example with 4 users

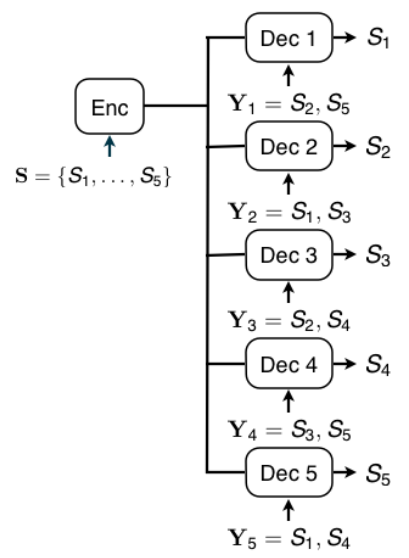

Fig. 5. Index coding example with 5 users

where $\theta(I, k)=0.5$ for all $|I|=3$ and $\theta(I, k)=1$ for all $|I|=4$. Now we move on to level-3 messages. Since there is only one level-4 message, $U_{1234}$, all possible excess bits at this stage will be moved to $U_{1234}$. We start with $U_{124}$. Since $H\left(U_{124} \mid \mathbf{Y}_{\mathbf{1}}\right)=1.5, H\left(U_{124} \mid \mathbf{Y}_{\mathbf{2}}\right)=1.5, H\left(U_{124} \mid \mathbf{Y}_{\mathbf{4}}\right)=2$, we have $i^{*}=1, j^{*}=4$. We declare, say, $S_{3}$ to be excess and we move all of $\theta(\{1,2,4\}, 3)$ to $U_{1234}$. Then we recalculate $H\left(U_{124} \mid \mathbf{Y}_{\mathbf{i}}\right)$, for $i \in\{1,2,4\}$. Now $i^{*}=2, j^{*}=1$ and the fraction of $S_{7}$, i.e., $\theta(\{1,2,4\}, 7)$, becomes an excess bit. We recalculate $H\left(U_{124} \mid \mathbf{Y}_{\mathbf{i}}\right)$, for $i \in\{1,2,4\}$ and all are equal. Hence we move on to another level-3 message, say $U_{134}$. For this message fraction of $S_{5}$ and $S_{9}$ become excess bits and are moved to $U_{1234}$. Lastly, all demands in $U_{123}, U_{234}$ are excess bits and moved to $U_{1234}$. This concludes Step 2.

Step 3: Since there is no XOR opportunities as described in this step, we only require to be 2 . Then the nonzero $\theta(I, k)$ 's are

$$
\begin{aligned}
& \theta(\{1,2,4\}, 13), \theta(\{1,2,4\}, 8), \theta(\{1,2,4\}, 4), \\
& \theta(\{1,3,4\}, 13), \theta(\{1,3,4\}, 10), \theta(\{1,3,4\}, 6), \\
& \theta([4], 1), \theta([4], 2), \theta([4], 11), \theta([4], 12), \theta([4], 3), \theta([4], 7), \\
& \theta([4], 5), \theta([4], 9), \theta([4], 6), \theta([4], 4), \theta([4], 8), \theta([4], 10),
\end{aligned}
$$

where $\theta(I, k)=0.5$ for $k \in\{4,6,8,10,13\}$ and the rest are 1 . As a result, the rate coming from level-3 and level-4 messages are 2 and 8.5 bits respectively and the total rate for this problem is 10.5 bits.

From the linear programming lower bound 5 stated in [5], we get 10.5 bits showing that S-CAPM is optimal.

Example 5. We consider the "5-cycle" index coding problem shown in Fig. 5] Its optimal rate is found in [5]. When we apply $S$-CAPM, we determine the messages and $t$ as follows:

Step 1 : After this step we have the following nonzero $\theta(I, k)$ 's. $\theta(\{1,2,5\}, 1), \theta(\{1,2,3\}, 2), \theta(\{2,3,4\}, 3), \theta(\{3,4,5\}, 4), \theta(\{1,4,5\}, 5)$, where all $\theta(I, k)=1$ for all $k \in[5]$.

${ }^{5}$ This lower bound is for the zero-error setting. However, it can be modified to handle vanishing block error probabilities. 
Step 2 : We start with level-3 messages. Note that all demands in level-3 messages are excess bits to be moved to level-4 messages. Since each leftover bit has two possible level-4 messages to go, $\theta(I, k)=0.5$ for all $k \in[5]$. Then the nonzero $\theta(I, k)$ 's are

$\theta(\{1,2,3,5\}, 1), \theta(\{1,2,3,5\}, 2)$,

$\theta(\{1,2,4,5\}, 1), \theta(\{1,2,4,5\}, 5)$,

$\theta(\{1,2,3,4\}, 2), \theta(\{1,2,3,4\}, 3)$,

$\theta(\{1,3,4,5\}, 4), \theta(\{1,3,4,5\}, 5)$,

$\theta(\{2,3,4,5\}, 3), \theta(\{2,3,4,5\}, 4)$.

Now, we move on to level-4 messages. Since there are no leftover bits at level-4 messages and all nonzero $\theta(I, k)=0.5$, we set $t=2$ concluding S-CAPM. Hence, the total rate becomes 2.5 bits which is the optimal rate.

\section{ACKNOWLEDGMENT}

This work was supported by Intel, Cisco, and Verizon under the Video-Aware Wireless Networks (VAWN) program.

\section{REFERENCES}

[1] Y. Birk and T. Kol, "Informed-source coding-on-demand (iscod) over broadcast channels," in INFOCOM '98. Seventeenth Annual Joint Conference of the IEEE Computer and Communications Societies. Proceedings. IEEE, vol. 3, 1998, pp. 1257-1264 vol.3.

[2] Z. Bar-Yossef, Y. Birk, T. S. Jayram, and T. Kol, "Index coding with side information," in Foundations of Computer Science, 2006. FOCS '06. 47th Annual IEEE Symposium on, 2006, pp. 197-206.

[3] K. Shanmugam, A. Dimakis, and M. Langberg, "Local graph coloring and index coding," in Information Theory Proceedings (ISIT), 2013 IEEE International Symposium on, July 2013, pp. 1152-1156.

[4] K. Shanmugam, A. G. Dimakis, and M. Langberg, "Graph theory versus minimum rank for index coding," in Information Theory (ISIT), 2014 IEEE International Symposium on, June 2014, pp. 291-295.

[5] A. Blasiak, R. Kleinberg, and E. Lubetzky, "Broadcasting with side information: Bounding and approximating the broadcast rate," Information Theory, IEEE Transactions on, vol. 59, no. 9, pp. 5811-5823, 2013.

[6] A. Tehrani, A. Dimakis, and M. Neely, "Bipartite index coding," in Information Theory Proceedings (ISIT), 2012 IEEE International Symposium on, July 2012, pp. 2246-2250.

[7] H. Maleki, V. Cadambe, and S. Jafar, "Index coding ;an interference alignment perspective," Information Theory, IEEE Transactions on, vol. 60, no. 9, pp. 5402-5432, Sept 2014

[8] F. Arbabjolfaei, B. Bandemer, Y.-H. Kim, E. Sasoglu, and L. Wang, "On the capacity region for index coding," in Information Theory Proceedings (ISIT), 2013 IEEE International Symposium on, July 2013, pp. 962-966.

[9] F. Arbabjolfaei and Y.-H. Kim, "Local time sharing for index coding," in Information Theory (ISIT), 2014 IEEE International Symposium on, June 2014, pp. 286-290.

[10] N. Alon, E. Lubetzky, U. Stav, A. Weinstein, and A. Hassidim, "Broadcasting with side information," in Foundations of Computer Science, 2008. FOCS '08. IEEE 49th Annual IEEE Symposium on, 2008, pp. 823-832.

[11] E. Lubetzky and U. Stav, "Nonlinear index coding outperforming the linear optimum," Information Theory, IEEE Transactions on, vol. 55, no. 8, pp. $3544-3551,2009$

[12] C. Heegard and T. Berger, "Rate distortion when side information may be absent," Information Theory, IEEE Transactions on, vol. 31, no. 6, pp. 727-734, 1985.

[13] A. H. Kaspi, "Rate-distortion function when side-information may be present at the decoder," Information Theory, IEEE Transactions on, vol. 40, no. 6, pp. 2031-2034, 1994

[14] R. Timo, T. Chan, and A. Grant, "Rate distortion with side-information at many decoders," Information Theory, IEEE Transactions on, vol. 57, no. 8, pp. 5240-5257, 2011.

[15] A. E. Gamal and Y.-H. Kim, Network Information Theory. New York, NY, USA: Cambridge University Press, 2012.

[16] E. Arikan, "Some complexity results about packet radio networks," Information Theory, IEEE Transactions on, vol. 30, no. 4, pp. 681-685, 1984.

[17] S. Vishwanath, G. Kramer, S. Shamai, S. Jafar, and A. Goldsmith, "Capacity bounds for Gaussian vector broadcast channels," DIMACS SERIES IN DISCRETE MATHEMATICS AND THEORETICAL COMPUTER SCIENCE, vol. 62, pp. 107-122, 2004.

[18] P. Vishwanath and D. Tse, "On the capacity of the multiple antenna broadcast channel," DIMACS SERIES IN DISCRETE MATHEMATICS AND THEORETICAL COMPUTER SCIENCE, vol. 62, pp. 87-106, 2004.

[19] S. Watanabe, "The rate-distortion function for product of two sources with side-information at decoders," Information Theory, IEEE Transactions on, vol. 59, no. 9, pp. 5678-5691, Sept 2013.

[20] R. Timo, A. Grant, and G. Kramer, "Lossy broadcasting with complementary side information," Information Theory, IEEE Transactions on, vol. 59, no. 1, pp. 104-131, 2013.

[21] S. Unal and A. B. Wagner, "Vector Gaussian rate-distortion with variable side information," in Information Theory Proceedings (ISIT), 2014 IEEE International Symposium on, 2014.

[22] A. Sgarro, "Source coding with side information at several decoders," Information Theory, IEEE Transactions on, vol. 23, no. 2, pp. 179-182, 1977.

[23] R. Timo, A. Grant, T. Chan, and G. Kramer, "Source coding for a simple network with receiver side information," in Information Theory Proceedings (ISIT), 2008 IEEE International Symposium on, 2008, pp. 2307-2311.

[24] R. Timo, T. J. Oechtering, and M. Wigger, "Source coding problems with conditionally less noisy side information," Information Theory, IEEE Transactions on, vol. 60, no. 9, pp. 5516-5532, 2014

[25] T. Laich and M. Wigger, "Utility of encoder side information for the lossless Kaspi/Heegard-Berger problem," in Information Theory Proceedings (ISIT), 2013 IEEE International Symposium on, 2013, pp. 3065-3069.

[26] M. Langberg and M. Effros, "Network coding: Is zero error always possible?" in Communication, Control, and Computing (Allerton), 2011 49th Annual Allerton Conference on, Sept 2011, pp. 1478-1485.

[27] S. Unal and A. B. Wagner, "A rate-distortion approach to index coding," in ITA. IEEE, 2014, pp. 1-5.

[28] M. Neely, A. Tehrani, and Z. Zhang, "Dynamic index coding for wireless broadcast networks," Information Theory, IEEE Transactions on, vol. 59, no. 11, pp. 7525-7540, Nov 2013.

[29] M. Maddah-Ali and U. Niesen, "Fundamental limits of caching," Information Theory, IEEE Transactions on, vol. 60, no. 5, pp. 2856-2867, May 2014.

[30] M. A. Maddah-Ali and U. Niesen, "Decentralized caching attains order-optimal memory-rate tradeoff," CoRR, vol. abs/1301.5848, 2013.

[31] R. T. Rockafellar, Convex Analysis. Princeton University Press, 1970. 


\section{APPENDIX}

Lemma 4. The minimum in (2) is unaffected by the presence of the cardinality bounds in condition 5).

Proof: Without loss of generality let $\sigma(i)=i$ for all $i \in[m]$. Let $P_{U_{1}, \ldots, U_{m}, S}\left(u_{1}, \ldots, u_{m}, s\right)$ denote the joint distribution of $\left(U_{1}, \ldots, U_{m}, S\right)$. We follow a procedure similar to that of [19]. First we find the bound on the cardinality of $U_{1}$, then $U_{2}$, etc.

To begin with, we consider the following $(|\mathcal{S}|-1)+1+m$ functions of $P_{U_{2}, \ldots, U_{m}, S \mid U_{1}}\left(., \ldots, . \mid u_{1}\right)$, denoted as $g_{s}^{0}, s \in|\mathcal{S}|-1$ and $g_{l_{0}}^{0}, g_{d_{1}}^{0}, \ldots, g_{d_{m}}^{0}$.

$$
g_{s}^{0}\left(P_{U_{2}, \ldots, U_{m}, S \mid U_{1}}\left(., \ldots, . \mid u_{1}\right)\right)=\sum_{u_{2}, \ldots, u_{m}} P_{U_{2}, \ldots, U_{m}, S \mid U_{1}}\left(u_{2}, \ldots, u_{m}, s \mid u_{1}\right),
$$

for $s=1, \ldots,|\mathcal{S}|-1$ and

$$
\begin{aligned}
& g_{l o}^{0}\left(P_{U_{2}, \ldots, U_{m}, S \mid U_{1}}\left(., \ldots, . \mid u_{1}\right)\right) \\
& =H\left(S \mid Y_{1}\right)-H\left(S \mid U_{1}=u_{1}, Y_{1}\right)+I\left(S ; U_{2} \mid U_{1}=u_{1}, Y_{1}, Y_{2}\right)+\cdots+I\left(S ; U_{m} \mid U_{1}=u_{1}, \ldots, U_{(m-1)}, Y_{1}, \ldots, Y_{m}\right),
\end{aligned}
$$

and

$$
\begin{aligned}
& g_{d_{1}}^{0}\left(P_{U_{2}, \ldots, U_{m}, S \mid U_{1}}\left(., \ldots, . \mid u_{1}\right)\right)=\mathbb{E}\left[d\left(S, g_{1}\left(u_{1}, Y_{1}\right)\right) \mid U_{1}=u_{1}\right] \\
& \vdots \\
& g_{d_{m}}^{0}\left(P_{U_{2}, \ldots, U_{m}, S \mid U_{1}}\left(., \ldots, \mid u_{1}\right)\right)=\mathbb{E}\left[d\left(S, g_{m}\left(U_{m}, Y_{m}\right)\right) \mid U_{1}=u_{1}\right]
\end{aligned}
$$

Then by Carathéodory's theorem [31, Theorem 17.1] we can find a random variable $U_{1}^{1}$ with $\left|U_{1}^{1}\right| \leq|\mathcal{S}|+m+1$ and random variables $U_{2}^{1}, \ldots, U_{m}^{1}$ where $P_{U_{1}^{1}, \ldots, U_{m}^{1}, S}\left(u_{1}, \ldots, u_{m}, s\right)=P_{U_{1}^{1}}\left(u_{1}\right) P_{U_{2}, \ldots, U_{m}, S \mid U_{1}}\left(u_{2}, \ldots, u_{m}, s \mid u_{1}\right)$ such that from (49) $P_{S}$ is preserved and from 50 )

$$
\begin{aligned}
& I\left(S ; U_{1}^{1} \mid Y_{1}\right)+I\left(S ; U_{2}^{1} \mid U_{1}^{1}, Y_{1}, Y_{2}\right)+\cdots+I\left(S ; U_{m}^{1} \mid U_{1}^{1}, \ldots, U_{(m-1)}^{1}, Y_{1}, \ldots, Y_{m}\right) \\
& =I\left(S ; U_{1} \mid Y_{1}\right)+I\left(S ; U_{2} \mid U_{1}, Y_{1}, Y_{2}\right)+I\left(S ; U_{m} \mid U_{1}, \ldots, U_{(m-1)}, Y_{1}, \ldots, Y_{m}\right),
\end{aligned}
$$

and we have

$$
\begin{aligned}
& \mathbb{E}\left[d\left(S, g_{1}\left(U_{1}^{1}, Y_{1}\right)\right]=\mathbb{E}\left[d\left(S, g_{1}\left(U_{1}, Y_{1}\right)\right)\right]\right. \\
& \vdots \\
& \mathbb{E}\left[d\left(S, g_{m}\left(U_{m}^{1}, Y_{1}\right)\right]=\mathbb{E}\left[d\left(S, g_{m}\left(U_{m}, Y_{1}\right)\right)\right] .\right.
\end{aligned}
$$

Now we consider the following $\left|U_{1}\right||\mathcal{S}|+(m-1)$ functions of $P_{U_{1}^{1}, U_{3}^{1}, \ldots, U_{m}^{1}, S \mid U_{2}^{1}}\left(., \ldots, . \mid u_{2}\right)$.

$$
g_{s}^{1}\left(P_{U_{1}^{1}, U_{3}^{1}, \ldots, U_{m}^{1}, S \mid U_{1}^{1}}\left(., \ldots, . \mid u_{2}\right)\right)=\sum_{u_{3}, \ldots, u_{m}} P_{U_{1}^{1}, U_{3}^{1}, \ldots, U_{m}^{1}, S \mid U_{2}^{1}}\left(., \ldots, . \mid u_{2}\right),
$$

for $\left(u_{1}, s\right)=1, \ldots,\left|U_{1}\right||\mathcal{S}|-1$ and

$$
\begin{aligned}
g_{l o}^{1} & \left(P_{U_{1}^{1}, U_{3}^{1}, \ldots, U_{m}^{1}, S \mid U_{2}^{1}}\left(., \ldots, . \mid u_{2}\right)\right) \\
= & -H\left(S \mid U_{1}^{1}, U_{2}^{1}=u_{2}, Y_{1}, Y_{2}\right)+I\left(S ; U_{3}^{1} \mid U_{1}^{1}, U_{2}^{1}=u_{2}, Y_{1}, Y_{2}, Y_{3}\right) \cdots \\
& +I\left(S ; U_{m}^{1} \mid U_{1}^{1}, U_{2}^{1}=u_{2}, U_{3}^{1} \ldots, U_{(m-1)}^{1}, Y_{1}, \ldots, Y_{m}\right),
\end{aligned}
$$

and

$$
\begin{aligned}
& g_{d_{2}}^{1}\left(P_{U_{1}^{1}, U_{3}^{1}, \ldots, U_{m}^{1}, S \mid U_{2}^{1}}\left(., \ldots, . \mid u_{2}\right)\right)=\mathbb{E}\left[d\left(S, g_{2}\left(u_{2}, Y_{2}\right) \mid U_{2}^{1}=u_{2}\right]\right. \\
& \vdots \\
& g_{d_{m}}^{1}\left(P_{U_{1}^{1}, U_{3}^{1}, \ldots, U_{m}^{1}, S \mid U_{2}^{1}}\left(., \ldots, . \mid u_{2}\right)\right)=\mathbb{E}\left[d\left(S, g_{m}\left(U_{m}^{1}, Y_{m}\right) \mid U_{2}^{1}=u_{2}\right] .\right.
\end{aligned}
$$

Again by Carathéodory's theorem, there is a random variable $U_{2}^{2}$ with $\left|U_{2}^{2}\right| \leq\left|U_{1}\right||\mathcal{S}|+m$ and random variables $U_{3}^{2}, \ldots, U_{m}^{2}$ where $P_{U_{1}^{1}, U_{2}^{2}, \ldots, U_{m}^{2}, S}\left(u_{1}, \ldots, u_{m}, s\right)$ is equal to $P_{U_{2}^{2}}\left(u_{2}\right) P_{U_{1}^{1}, U_{3}^{1}, \ldots, U_{m}^{1}, S \mid U_{2}^{1}}\left(u_{1}, u_{3}, \ldots, u_{m}, s \mid u_{2}\right)$ such that $P_{U_{1}^{1} S}$ is preserved (from 51).

Since $P_{U_{1}^{1} S}$ is preserved, $\mathbb{E}\left[d\left(S, g_{2}\left(U_{1}^{1}, Y_{1}\right)\right], H\left(S \mid U_{1}^{1}, Y_{1}, Y_{2}\right)\right.$, and $I\left(S ; U_{1}^{1} \mid Y_{1}\right)$ are preserved. Also, from 52 we have

$$
\begin{aligned}
& I\left(S ; U_{1}^{1} \mid Y_{1}\right)+I\left(S ; U_{2}^{2} \mid U_{1}^{1}, Y_{1}, Y_{2}\right)+\cdots+I\left(S ; U_{m}^{2} \mid U_{1}^{1}, U_{2}^{2}, \ldots, U_{(m-1)}^{2}, Y_{1}, \ldots, Y_{m}\right) \\
& =I\left(S ; U_{1}^{1} \mid Y_{1}\right)+I\left(S ; U_{2}^{1} \mid U_{1}^{1}, Y_{1}, Y_{2}\right)+I\left(S ; U_{m}^{1} \mid U_{1}^{1}, \ldots, U_{(m-1)}^{1}, Y_{1}, \ldots, Y_{m}\right) .
\end{aligned}
$$


Lastly, we have the following equalities.

$$
\begin{aligned}
& \mathbb{E}\left[d\left(S, g_{2}\left(U_{2}^{2}, Y_{2}\right)\right]=\mathbb{E}\left[d\left(S, g_{2}\left(U_{2}^{1}, Y_{2}\right)\right)\right]\right. \\
& \vdots \\
& \mathbb{E}\left[d\left(S, g_{m}\left(U_{m}^{1}, Y_{m}\right)\right]=\mathbb{E}\left[d\left(S, g_{m}\left(U_{m}^{1} Y_{m}\right)\right)\right] .\right.
\end{aligned}
$$

By applying the above procedure to $U_{3}^{2}, \ldots, U_{m}^{2}$ consecutively and relabeling $\left(U_{1}^{1}, U_{2}^{2}, \ldots\right)$ as $\left(U_{1}, \ldots, U_{m}\right)$ we obtain the cardinality bounds as stated in the condition 5) of Theorem 1 . 